\title{
Bounds on Renormalized Feynman Graphs
}

\author{
J. Feldman`, J. Magnen, V. Rivasseau, and R. Sénéor \\ Centre de Physique Théorique de l'Ecole Polytechnique, Plateau de Palaiseau, \\ F-91128 Palaiseau Cedex, France
}

\begin{abstract}
We prove two bounds on the value of renormalized Euclidean Feynman graphs. One is a relatively crude but widely applicable bound; the other a finer bound applicable to $\phi_{4}^{4}$-like models.
\end{abstract}

\section{Introduction}

In [FMRS 1] we used a phase space expansion to prove an exponential bound on completely convergent Euclidean Feynman graphs:

$$
|G| \leqq K^{L(G)},
$$

where $|G|$ is the value of the amplitude associated to the graph $G$ and $L(G)$ is the number of lines in the graph. In this paper $K$ will stand for various numerical constants whose precise values are largely irrelevant.

In this companion paper we apply the same expansion to the technically more difficult cases of renormalized graphs.

The bound (1.1) fails for renormalized graphs in strictly renormalizable theories; explicit families of graphs with factorial growth have been constructed [GN1, L]. On the other hand in [CR], a general bound on graphs of massive $\phi_{4}^{4}$ has been derived, which grows as the factorial of the number of renormalization subtractions. It extends to renormalized graphs of massless $\phi_{4}^{4}$ at non-exceptional momenta [CPR]. These bounds are not optimal.

For example, if

Fig. 1.1

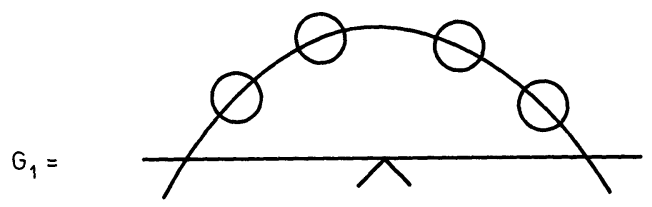

* Permanent address: Department of Mathematics, University of Britisch Columbia, Vancouver B.C. V6T 1Y4, Canada 
with $n$ mass bubbles, at zero external momenta, the bound of [CR] is $\left|G_{1}\right| \leqq($ const)(2n)!. We prove two bounds on renormalized graphs. In Sect. II, we prove a bound which is very widely applicable but quite crude. Applied to the testgraph $G_{1}$ it gives $\left|G_{1}\right| \leqq($ const)( $3 n)$ !. In Sect. III, we prove a finer bound which is applicable only to $\phi_{4}^{4}$-like models, which gives $\left|G_{1}\right| \leqq($ const $) n$ !, the correct behavior. " $\phi_{4}^{4}$-like models" means essentially that for $m \geqq 5$ the connected $m$-point functions are superficially convergent, and for $m=3,4$, they are at worst logarithmically divergent. Fermions are allowed, so the (Gross-Neveu) ${ }_{2}$ model [GN 1] falls into this class. Therefore we recover in particular all the results of [CR].

The advantage of the methods used in this paper over the original techniques of $[\mathrm{CR}]$ is that they are directly applicable in the larger context of constructive field theory [FMRS3].

We now provide an outline of the expansion used in [FMRS1] or [FMRS2]. Consider a Euclidean Feynman graph represented in position space. Bound each propagator by:

$$
\begin{aligned}
C(x, y) & =\left|\int \frac{d^{d} k}{(2 \pi)^{d}} \frac{e^{i k(x-y)}}{\left(k^{2}+m^{2}\right)^{p}}\right| \\
& \leqq K \sum_{i=0}^{\infty} M^{i \delta} \exp \left(-M^{i}|x-y|\right),
\end{aligned}
$$

where $\delta=d-2 p$, and assume $m>0, M$ big enough (here one should take $M \geqq 12$, with some straightforward changes one can take any $M>1$ ). We fix one value $i_{\ell}$ of $i$ for each propagator $\ell$ (a "momentum assignment"). To perform the integral over position space, let us slightly simplify the recipe of [FMRS 1, FRMS 2]. ${ }^{1}$

We make repeated use of: $\int d^{d} x \exp \left(-M^{i}|x-y|\right)=K M^{-i d}$.

Any spanning tree $T$ of $G$ can be used to define a partial order on the vertices of $G$ (choosing some external vertex of $G$ to be the biggest). Then integrating over the positions of the vertices in that order, starting from the smallest ones, one gets:

$$
\begin{aligned}
\int \prod_{v} d^{d} x_{v} \prod_{\ell \in G} \exp \left(-M^{i}\left|x_{\ell}-y_{\ell}\right|\right) & \leqq \int \prod_{v} d^{d} x_{v} \prod_{\ell \in T} \exp \left(-M^{i_{\ell}} \mid x_{\ell}-y_{\ell}\right) \\
& =\prod_{v} K M^{-i_{v} d},
\end{aligned}
$$

where $i_{v}$ is the scale of the line directly above the vertex:

Fig. 1.2

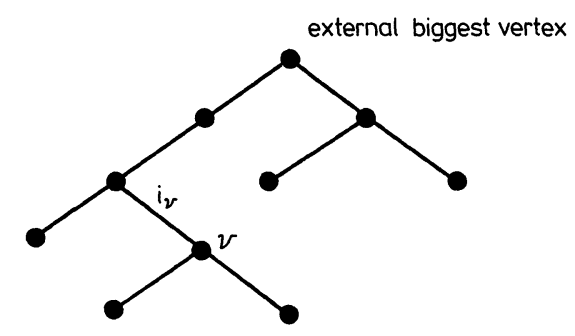

We must choose $T$ so as to make the $i_{v}$ 's as large as possible. Let $G_{i}$ be the subgraph of $G$ made of these lines $\ell$ with index $i_{\ell} \geqq i$. $G_{i}$ is the union of connected components $G_{i}^{k}$. We choose $T$ so that its restriction to every $G_{i}^{\alpha}$ is still a spanning tree in $G_{i}^{\alpha}$. This

1 We thank G. Felder for this simplification 
is possible since the $G_{i}^{\alpha}$ s form a forest of connected graphs (build inductively, starting from the smallest $G_{i}^{\alpha}$ s).

We claim that for this choice of $T$, and for any fixed $G_{j}^{\alpha}$ every vertex $v$, save one, of $G_{j}^{\alpha}$ has $i_{v} \geqq j$. (This is an easy consequence of the connectedness of $T \cap G_{j}^{\alpha}$.)

Hence, aside from irrelevant constants, our graph is bounded by the sum over momentum assignments of

$$
\prod_{j \geqq 1} \prod_{k}\left\{\left[\prod_{\ell \in G_{j}^{k}} M^{\delta(\ell)}\right]^{-d\left(V\left(G_{j}^{k}\right)-1\right)}\right\}=\prod_{j \geqq 1} \prod_{k} M^{D\left(G_{j}^{k}\right)},
$$

where $V\left(G_{j}^{k}\right)$ is the number of vertices, and $D\left(G_{j}^{k}\right)$ the superficial degree of divergence of $G_{j}^{k}$. Assume now that there is an $\varepsilon>0$ (depending on the model) such that $D\left(G_{j}^{k}\right) \leqq-\varepsilon E\left(G_{j}^{k}\right)$, where $E\left(G_{j}^{k}\right)$ is the number of external lines of $G_{j}^{k}$. Let us achieve the sum over momentum assignments when the maximum number of lines that may be hooked to a vertex is bounded (as in $\phi_{4}^{4}$-like models for instance). For the general case we refer to [FMRS1].

We write

$$
\text { (1.4) } \leqq \prod_{j \geqq 1} \prod_{k} M^{-\varepsilon E\left(G_{j}^{h}\right)} \leqq \prod_{v}\left\{\prod_{\ell, \ell^{\prime} \text { hooked to } v} M^{-\varepsilon^{\prime} \mid i_{\ell}-i_{\ell}^{\prime} \prime}\right\},
$$

where $\varepsilon^{\prime}$ depends on $\varepsilon$ and on the maximal number of lines per vertex.

We have now an exponential decay in momentum space between lines hooked to a common vertex, dual to the usual exponential decay in position space between vertices linked by a line. We order the lines of $G$ as $\ell_{1}, \ldots, \ell_{n}, \ldots, \ell_{L(G)}$, such that $\ell_{1}$ is hooked to an external vertex and each $\left\{\ell_{1}, \ldots, \ell_{n}\right\}, n=1, \ldots, L(G)$ is connected.

Then one has

$$
(1.5) \leqq \prod_{n=1}^{L(G)} M^{-\varepsilon^{\prime}\left|i_{n^{\prime}(n)}-i_{\ell_{n}}\right|}
$$

with $n^{\prime}(n)<n$, and, by convention, $i_{\ell_{0}}=0$. Applying

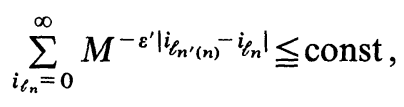

starting with (1.6) and working backwards completes the proof of (1.1).

Notice that if $G$ contains unrenormalized superficially divergent subgraphs, we can have $D\left(G_{j}^{k}\right) \geqq 0$, in (1.4) and the exponential decay in momentum space disappears, or even becomes exponential growth.

It is that problem which is tackled by renormalization in the next sections.

In the course of completing this work, we received preprints from Gallavoti and Nicolò [GN2] and Gallavoti [G] in which the factorial bounds of [CR] are also derived by a phase space analysis, using an original and powerful formalism. This formalism is not aimed at the study of individual and rather general Feynman graphs, in contrast with the present paper.

\section{Renormalized Graphs: Bounds for General Models}

We assume the reader's familiarity with some basic notions of Feynman graph theory, like trees, forests, superficial degree of divergence, etc.... We shall be considering connected graphs $G$, viewed in position space as usual, whose lines are 
the propagators of massive, power law fields. In other words we will not consider logarithmic propagators in this paper. We start by describing how we shall renormalize these graphs. We use two variants of Zimmermann's forest scheme. Before integrating over the positions of the internal vertices we apply the renormalization operator

$$
\mathfrak{R}=\sum_{\mathfrak{F}} \prod_{g \in \mathfrak{F}}\left(-\mathfrak{C}_{g}\right) .
$$

Here the $\mathfrak{F}$ 's are forests of divergent subgraphs of $G ; \mathfrak{C}_{g}$ integrates over the positions of the internal vertices of the subgraph and replaces the resulting function of $\left\{x_{v} \mid v \in \mathfrak{B}_{E}(g)\right\}$ by the distribution supported at coinciding arguments which is, in momentum space, the Taylor polynomial of degree $D(g)$ for $g$ (a precise formula will be given shortly); the Taylor operators $\Pi\left(-\mathfrak{C}_{g}\right)$ for convenience are ordered so that if $g_{1} \subset g_{2},-\mathfrak{C}_{g_{1}}$ is applied before $-\mathfrak{C}_{g_{2}}$.

In the variant we use in this section we shall sum over the set $\mathfrak{F}^{D}(G)$ of all connected divergent forests of $G$ (including $\mathfrak{F}=\emptyset$ ). This means that every $g \in \mathfrak{F}$ must be a connected, superficially divergent subgraph of $G$. This leads to a bound which may be applied to very general graphs and whose proof is, relatively speaking, not clouded by technical detail. On the other hand the bound is relatively crude. It grows as $S^{D}(G)$ !, where the number of subtractions $S^{D}(G)$ in $G$ is defined by

$$
S^{D}(G)=\sup _{\mathscr{F} \in \mathfrak{F}^{D(G)}}|\mathfrak{F}| .
$$

In the variant we use in Sect. III, we shall consider only $\phi_{4}^{4}$-like models and we shall sum over the set $\mathfrak{F}^{C}(G)$ of all closed, proper divergent forests of $G$ (including $\mathfrak{F}=\emptyset$ ). Hence in this case every $g \in \mathfrak{F}$ must be a 1 PI, closed, divergent subgraph of $G$. A divergent subgraph $g \subseteq G$ is closed [CR] if and only if there does not exist a divergent subgraph $g^{\prime}$ obeying (1) $g \varsubsetneqq g^{\prime} \cong G$, (2) every external vertex of $g^{\prime}$ is also an external vertex of $g$. In the example below $g_{1}$ is closed but $g_{2}$ is not.

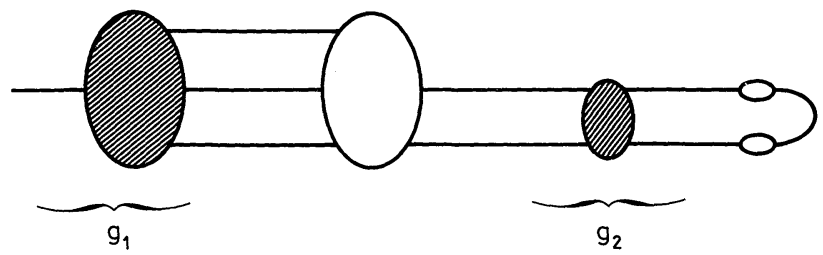

Fig. 2.1

Because we tailor everything to $\phi_{4}^{4}$ in this case we achieve a growth rate of $S^{C}(G)$ !, where

$$
S^{C}(G)=\sup _{\mathbb{F}_{\mathfrak{F}} \mathfrak{F}^{C}(G)}|\mathfrak{F}| .
$$

For example if there are $n$ mass bubbles in the graph $G_{1}$ of Fig. 1.1 we have $S^{C}(G)=n$, whereas $S^{D}(G)=3 n-1$.

We come to the precise definition of $\mathfrak{C}_{g}$. After integration of its internal vertices $g$ is a function $g\left(x_{1}, \ldots, x_{V_{E}}\right)$ of its $V_{E}$ external vertices. By translation invariance its Fourier transform is of the form $\delta\left(k_{1}+\ldots+k_{V_{E}}\right) \tilde{g}\left(k_{1}, \ldots, k_{V_{E}-1}\right)$. The Fourier 
transform of $\mathfrak{C}_{g} g$ is then simply $\delta\left(k_{1}+\ldots+k_{V_{E}}\right)$ times the Taylor polynomial

$$
\left.\sum_{j=0}^{D[G]} \frac{1}{j !} \frac{d^{j}}{d t^{j}} \tilde{g}\left(t k_{1}, \ldots, t k_{V_{E}-1}\right)\right|_{t=0} .
$$

Here $D[G]$ is the largest integer less than or equal to $D(G)$. It appears superficially that $k_{V_{E}}$ plays a distinguished role here. That is of course not the case thanks to translation invariance. In practice we will want to apply $\mathfrak{C}_{g}$ to the test functions smearing $g$ rather than to the unsmeared value of $g$ itself, i.e. to use

$$
\begin{aligned}
& \int\left(\mathfrak{C}_{g} g\right)\left(x_{1}, \ldots, x_{v_{E}}\right) a\left(x_{1}, \ldots, x_{v_{E}}\right) d x_{1} \ldots d x_{v_{E}} \\
& \quad=\int g\left(x_{1}, \ldots, x_{v_{E}}\right)\left(\mathfrak{C}_{g}^{*} a\right)\left(x_{1}, \ldots, x_{v_{E}}\right) d x_{1} \ldots d x_{v_{E}} .
\end{aligned}
$$

Because $\mathfrak{C}_{g}$ is defined only for translation invariant $g$ 's it does not have a singlevalued adjoint. However (2.5) is nonetheless true for $\mathfrak{C}_{g}^{*}$ defined by:

$$
\left(\mathfrak{C}_{g}^{*} a\right)\left(x_{1}, \ldots, x_{v_{E}}\right)=\sum_{j=0}^{D[G]} \frac{1}{j !} \frac{d^{j}}{d t^{j}} a\left(x_{1}(t), x_{2}(t), \ldots, x_{v_{E}-1}(t),\left.x_{v_{E}}\right|_{t=0},\right.
$$

where $x_{i}(t)=x_{v_{E}}+t\left(x_{i}-x_{v_{E}}\right)$ for $i=1, \ldots, v_{E}-1$. Here $v_{E}$ really does play a distinguished role. However we could in (2.6) replace $v_{E}$ by any other external vertex of $g$, thereby defining a different $\mathfrak{C}_{g}^{*}$ but one for which (2.5) is still true.

Here is a simple example that illustrates how renormalization works in the context of the phase space expansion.

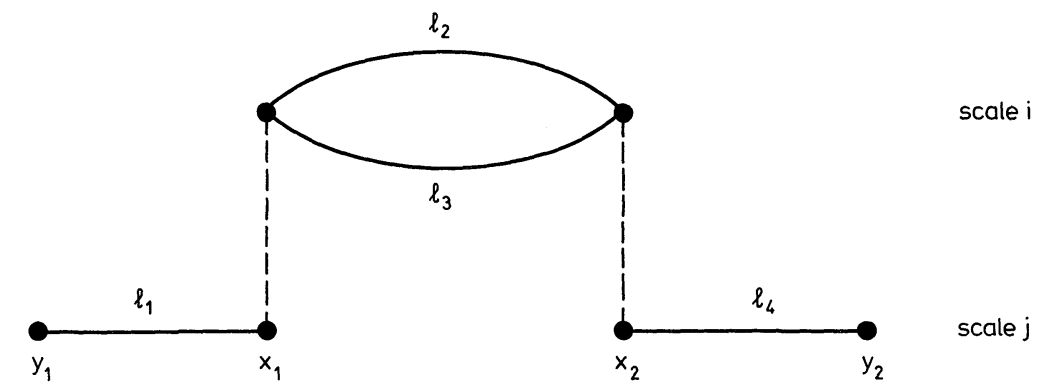

Fig. 2.2

The propagator in scale $k$, being the energy $M^{k}$ part of a $d=4$ boson propagator behaves essentially like $M^{2 k} \exp \left(-M^{k}|\ell|\right)$. Once we have done the integration over position space we will have associated with the subgraph momentum factor $M^{2 i} \times M^{2 i} \times M^{-4 i}=M^{0}$ [one $M^{2 i}$ from each propagator and $M^{-4 i}$ from the "non-fixed" vertex of $\longrightarrow$ see (1.4)]. This is not surprising since the subgraph has degree 0 . It is nonetheless distrastrous because there is no decay in $i$ to allow us to sum over the momentum assignment $i$. Suppose we now renormalize $\bigcirc$, i.e. apply $1-\mathfrak{C}_{g}$ to $\bigcirc$, i.e. apply $\left(1-\mathfrak{C}_{g}\right)^{*}$ to

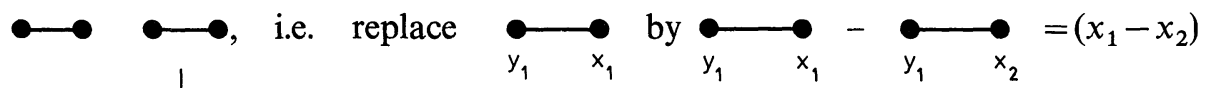
$\left.\cdot \nabla_{x} \bullet_{y_{1}} \rightarrow\right|_{\text {some } x}$. Then the gradient applied to $\exp \left(-M^{j}\left|x-y_{1}\right|\right)$ produces an 
extra factor of $M^{j}$, and using

$$
\left|x_{1}-x_{2}\right| \exp \left(-2 M^{i}\left|x_{1}-x_{2}\right|\right) \leqq M^{-i} \exp \left(-1.5 M^{i}\left|x_{1}-x_{2}\right|\right)
$$

$\left(x_{1}-x_{2}\right)$ produces an extra $M^{-i}$. Hence altogether renormalization gives us $M^{-(i-j)}$ : exponential decay between the momentum scale of the subgraph renormalized and the scale of its external lines. It is also worth noting that the bubble $g=\longrightarrow$ occurs as a connected component of a $G_{j}$ if and only if

$$
i_{g} \equiv \min \left\{i_{\ell_{2}}, i_{\ell_{3}}\right\}>\max \left\{i_{\ell_{1}}, i_{\ell_{4}}\right\} \equiv e_{g}
$$

Hence the divergence of - arises completely from momentum assignments respecting this inequality and consequently could be successfully cancelled by a counterterm of the form

$$
\int d x d y \sum_{i_{g}>e_{g}} \times \overbrace{i_{\ell_{3}}}^{i_{\ell_{2}}} \text { y } \varphi_{i_{\ell_{1}}}(x) \varphi_{i_{\ell_{4}}}(x)
$$

where $\varphi_{i}$ is the field of scale $i$. But this is not local because of the restriction $i_{g}>e_{g}$, and consequently is not a legitimate counterterm. Hence to preserve the locality of the theory we must, in addition to applying $\left(1-\mathfrak{C}_{g}\right)$ to $\sum_{i_{g}>e_{g}} \longrightarrow$, add the term $-\mathscr{C}_{g} \sum_{i_{g} \leqq e_{g}}$. Now $i_{g} \leqq e_{g}$ in effect put an ultraviolet cutoff on the bubble $\int d y \sum_{\substack{i_{2}, i_{3} \\ \text { such that } i_{g} \leqq e_{g}}} \times \overbrace{i_{3}}$, rendering it finite. It is bounded by const $e_{g}$. This $e_{g}$ is easily controlled by the $M^{-2 e_{g}}$ in $\sum_{i_{\ell_{1}}, i_{\ell_{4}}}^{\ell_{1}} \stackrel{\ell_{4}}{\longrightarrow}$, so the extra term is finite. However, in larger graphs there can be an accumulation of $e_{g}$ 's resulting in the production of factorials through sums of the form

$$
\sum_{i=0}^{\infty} M^{-\varepsilon i} i^{n} \sim \operatorname{const}^{n} n ! \text {. }
$$

This is the sole source of the factorials $S^{D}(G)$ ! and $S^{C}(G)$ !. We now state the hypothesis for our first theorem.

(H 2.1) The lines. Each line $\ell$ of the connected graph $G$ has a propagator $C_{\ell}$ which can be decomposed into

$$
C_{\ell}(x, y)=\sum_{j=0}^{\infty} C_{\ell, j}(x-y)
$$

with all $|n|^{\text {th }}$ order partial derivatives (we use the usual multi-index notation) obeying

$$
\left|\frac{\partial^{n}}{\partial x^{n}} C_{\ell, j}(x)\right| \leqq K^{\prime} e^{n^{2-v}} M^{j(\delta(\ell)+|n|)} e^{-M^{j}|x|}
$$

for some constants $M>1, K^{\prime}, v>0$ independent of $\ell$ and $\delta(\ell)$. Propagators are allowed to be matrix valued. 
Remark. (2.8) is obeyed by the propagator $1 /\left(k^{2}+m^{2}\right)^{p}$ with $m>1$ (we can always scale to achieve this), $\delta=d-2 p$, and

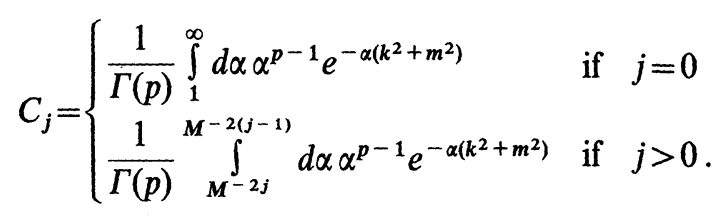

(H 2.2) The Vertices. There is a constant $\bar{L}$ such that at most $\bar{L}$ lines are hooked to any one vertex. [This is not essential (see FMRS1) but simplifies.]

(H 2.3) The External Vertices. The renormalized graph is integrated against a "function" $f(\mathbf{x})$ with $\mathbf{x}=\left(x_{v_{1}}, \ldots, x_{v_{V_{E}}}\right), \mathfrak{B}_{E}=\left\{v_{1}, \ldots, v_{V_{E}}\right\}$. For example to get the value of the graph in momentum space with momentum $k_{v}$ entering the external vertex $v$ (with $\sum_{v \in \mathfrak{B}_{E}} k_{v}=0$ ) one chooses

$$
f(\mathbf{x})=\left(\prod_{\substack{v \in \mathfrak{G}_{E} \\ v \neq v_{v_{E}}}} e^{i k_{v} x_{v}}\right) \delta\left(x_{v_{v_{E}}}\right) .
$$

(H 2.4) Power Counting. We assume that there exist constants such that for every connected subgraph $g \subseteq G$

$$
\begin{aligned}
& D(g) \leqq\left\{\begin{array}{ccc}
\bar{D} & \text { when } & D(g) \geqq 0 \\
-2 \varepsilon E(g) & \text { when } & D(g)<0,
\end{array}\right. \\
& E(g) \leqq \bar{E} \text { if } \quad D(g) \geqq 0 .
\end{aligned}
$$

In effect $\bar{D}$ and $\bar{E}$ are the maximal degree of divergence and maximal number of external lines for divergent subgraphs. For non-integral positive $D(g)$ we also assume $D[g]+1 \geqq D(g)+\varepsilon$.

(H 2.5) Divergent Forests. There is a constant $K^{\prime \prime}$ such that the number $\left|\mathfrak{F}^{D}(G)\right|$ of connected divergent forests of $G$ is bounded by $K^{\prime \prime L(G)}$.

Theorem 2.1. If hypotheses $(\mathrm{H} 2.1)-(\mathrm{H} 2.5)$ are satisfied, then for any $0<\zeta<1$ and any $v_{G} \in \mathfrak{B}_{E}(G)$, there exists a constant $K$ such that

$$
\left|\int f(\mathbf{x})(\Re G)(\mathbf{x}) d \mathbf{x}\right| \leqq K^{L(G)} S^{D}(G) ! \sup _{\mathbf{j} \in T(G)}\left\|\left(\prod_{v \in \mathfrak{B}_{E}(G)} \nabla_{x_{v}}^{j_{v}}\right) f\right\|_{G, v_{G}},
$$

where

$$
\begin{aligned}
\|f\|_{G, v_{G}} & =\int d \mathbf{x}\left[\sup _{\left\{x_{v} \mid v \in \mathfrak{B}_{I}(G)\right\}} \prod_{\ell \in G} \exp \left(-(1-\zeta) \mid x_{\ell}-y_{\ell}\right)\right] \bar{f}(\mathbf{x}), \\
\bar{f}(\mathbf{x}) & =\sup \left\{\left|f\left(\mathbf{x}^{\prime}\right)\right|:\left|x_{v}^{\prime}-x_{v}\right| \leqq\left[\begin{array}{ll}
0 & v=v_{G} \\
1 & v \neq v_{G}
\end{array}\right\},\right.
\end{aligned}
$$

and $T(G)$ gives the sets of differential operators acting on $f$ that can arise during the renormalization process. It obeys:
(i) For each $v \in \mathfrak{B}_{E}(G), 0 \leqq j_{v} \leqq \sup _{\mathcal{F}_{\in} \in \mathscr{F}^{D}(G)} \sum_{v \in g \in \mathscr{F}}(D[g]+1)$,
(ii) $|\mathbf{j}|=\sum_{v} j_{v} \leqq \sup _{\mathscr{F} \in \mathscr{F}^{D}(G)} \sum_{g \in \mathscr{F}}(D[g]+1)$,
(iii) $j_{v_{G}}=0$. 
Remark. If (H 2.5) fails, we still get the above bound with $S^{D}(G)$ ! replaced by $\sum_{\mathfrak{F} \in \mathfrak{\Im}^{D}(G)}$.

Comment. The Meaning of It All. Renormalization subtractions are effected by applying $1-\mathfrak{C}_{g}^{*}$ to the test function $f$ that is smearing $g$. By Taylor's theorem this results in the application of $D(g)+1$ derivatives to the test function. Hence (i), (ii).

Even for a graph without divergent subgraphs $G(\mathbf{x})$ need not be uniformly bounded thanks to singularities at coinciding arguments. However we may get something uniformly bounded by fixing any one external vertex $v_{G}$ of $G$ and smearing all the remaining external vertices. This smearing may be achieved as follows. For each $v \neq v_{G}$ pick a smearing function $\eta_{v}$ obeying

$$
\begin{aligned}
& \eta_{v}\left(x_{v}\right) \geqq 0, \\
& \int \eta_{v}\left(x_{v}\right) d x_{v}=1, \\
& \operatorname{supp} \eta_{v} \subset\left\{x_{v}|| x_{v} \mid \leqq r_{v}\right\} .
\end{aligned}
$$

For example $\eta_{v}$ could be the characteristic function of a ball of volume 1 . Then apply

$$
\begin{aligned}
& \left|\int \prod_{v} d x_{v}^{\prime} G\left(\mathbf{x}^{\prime}\right) f\left(\mathbf{x}^{\prime}\right)\right| \\
& \quad=\mid \int \prod_{v} d x_{v}^{\prime} d x_{v} G\left(\mathbf{x}^{\prime}\right) \delta\left(x_{v_{G}}-x_{v_{G}}^{\prime}\right) \prod_{v \neq v_{G}} \eta_{v}\left(x_{v}^{\prime}-x_{v}|f(\mathbf{x})|\right. \\
& \quad \leqq \int \prod_{v} d x_{v}^{\prime} d x_{v}\left|G\left(\mathbf{x}^{\prime}\right)\right| \delta\left(x_{v_{G}}^{\prime}-x_{v_{G}}\right) \prod_{v \neq v_{G}} \eta_{v}\left(x_{v}^{\prime}-x_{v}\right) \bar{f}(\mathbf{x}),
\end{aligned}
$$

where

$$
\begin{aligned}
\bar{f}(\mathbf{x}) & =\sup \left\{|f(\mathbf{x})|:\left|x_{v}^{\prime}-x_{v}\right| \leqq \begin{cases}0 & v=v_{G} \\
r_{v} & v \neq v_{G}\end{cases} \right. \\
& \equiv \int \Pi d x_{v}(m G)(\mathbf{x}) \bar{f}(\mathbf{x}) .
\end{aligned}
$$

$(m G)(\mathbf{x})$ now has each vertex except the fixed vertex $v_{G}$ smeared by $\eta_{v}$ at the cost of having to take local suprema of $f$. Hence the $v_{G}$ and the local suprema. Furthermore we can choose $v_{E}$ of $(2.6)$ so that no $\mathfrak{C}_{g}^{*}$ applies a derivative to $v_{G}$. Hence (iii).

By way of an example suppose $f(\mathbf{x})=\delta\left(x_{v_{V_{E}}}\right) \prod_{v \neq v_{V_{E}}} e^{i k_{v} x_{v}}$. We should choose $v_{G}=v_{E}$ to avoid both applying derivatives to the $\delta$-function and evaluating $\sup _{\left|x_{V_{E}}^{\prime}-x_{v_{V_{E}}}^{\prime}\right| \leqq 1} \delta\left(x_{v_{V_{E}}}^{\prime}\right)$. Each derivative $\nabla x_{v}$ simply brings down a $\mathbf{k}_{v}$. Hence our bound has a dependence on the external momentum which grows at worst like $\left[\max _{v}\left|\mathbf{k}_{v}\right|\right]^{\Sigma(D(g)+1)}$, with the sum running over $g \in \mathfrak{F}^{D}(G)$ such that
$\mathfrak{Y}_{E}(G) \cap g \neq \emptyset \square$

Proof of Theorem 2.1. The first order of business is to reorganize the renormalization operator $\mathfrak{R}$ of $(2.1)$ so as to perform renormalization cancellations when they are needed. We call this reorganization "classification of forests."

Classification of Forests. Divergent forests enter the problem of bounding $G$ in two somewhat independent ways. Firstly the definition (2.1) of the renormalization 
operator introduces a sum over all connected divergent forests of $G$. Secondly given an assignment $\mu$ of momentum scales to the lines of $G$, the bounding process of Sect. I naturally generates a forest of "dangerous" subgraphs of $G$ : the connected divergent subgraphs of

$$
G_{j}(\mu)=\left\{\ell \in G \mid i_{\ell} \geqq j\right\},
$$

where $i_{\ell}$ is the momentum scale of the line $\ell$.

The situation here is a little different from that of Sect. I since the Taylor operators $-\mathfrak{C}_{h}$ inhibit vision across the boundary of $h$. Hence in deciding whether or not $g \in \mathfrak{F}$ is dangerous, we should consider only those internal lines of $g$ that are not in

$$
A_{\mathfrak{F}}(g)=\bigcup_{\mathscr{F} \ni h € g g} h,
$$

and those external lines of $g$ that are in $B_{\Re}(g)$, the smallest subgraph of $₹$ strictly containing $g$. If no such subgraph exists we define $B_{\mathfrak{f}}(g)=G$. So given any assignment $\mu$ of momenta and any forest $\mathfrak{F} \in \mathfrak{F}^{D}(G)$ we define the sets of dangerous subgraphs of $\mathfrak{F}$ and safe subgraphs of $\mathfrak{F}$, denoted $\mathfrak{D}_{\mu}(\mathfrak{F})$ and $\mathfrak{S}_{\mu}(\mathfrak{F})$ respectively ${ }^{2}$ by:

$$
\begin{aligned}
g \in \mathfrak{D}_{\mu}(\mathfrak{F}) \Leftrightarrow & g \in \mathfrak{F}, g / A_{\mathfrak{F}}(g) \text { is a connected component of } \\
& \left(B_{\mathfrak{F}}(g) / A_{\mathfrak{F}}(g)\right)_{j}(\mu) \text { for some } j \\
\Leftrightarrow & g \in \mathfrak{F}, i_{g}(\mathfrak{F})>e_{g}(\mathfrak{F}),
\end{aligned}
$$

where

$$
\begin{aligned}
& i_{g}(\mathfrak{F})=\min \left\{i_{\ell} \mid \ell \in g-A_{\mathscr{F}}(g)\right\}, \\
& e_{g}(\mathfrak{F})=\max \left\{i_{\ell} \mid \ell \in E(g) \cap B_{\mathfrak{F}}(g)\right\},
\end{aligned}
$$

and

$$
g \in \mathfrak{S}_{\mu}(\mathfrak{F}) \Leftrightarrow g \in \mathfrak{F}, \quad i_{g}(\mathfrak{F}) \leqq e_{g}(\mathfrak{F}) .
$$

We remind the reader that $E(g)$ is the set of external lines of $g . E(g) \cap B_{\mathfrak{F}}(g)$ can be empty only when $g=G$; then we define $e_{g}=0$. It is obvious that if $\mathfrak{F}_{1}$, $\mathfrak{F}_{2} \in \mathfrak{F}^{\mathbb{D}}(G), \mathfrak{F}_{1} \cong \mathfrak{F}_{2}$, then:

$$
\begin{aligned}
& i_{g}\left(\mathfrak{F}_{1}\right) \leqq i_{g}\left(\mathfrak{F}_{2}\right), \\
& e_{g}\left(\mathfrak{F}_{1}\right) \geqq e_{g}\left(\mathfrak{F}_{2}\right),
\end{aligned}
$$

for all $g \in \mathfrak{F}_{1}$. From the definition of $\mathfrak{D}_{\mu}(\mathfrak{F})$ it is also true that:

\section{Lemma 2.2.}

$$
\begin{aligned}
i_{g}(\mathfrak{F}) & =i_{g}\left(\mathfrak{\Im}_{\mu}(\mathfrak{F}) \cup\{g\}\right), \\
e_{g}(\mathfrak{F}) & =e_{g}\left(\mathfrak{\Im}_{\mu}(\mathfrak{F}) \cup\{g\}\right) .
\end{aligned}
$$

This lemma will be proved later. Consequently $\mathfrak{S}_{\mu}\left(\mathfrak{S}_{\mu}(\mathfrak{F})\right)=\mathfrak{\Xi}_{\mu}(\mathfrak{F})$, so $\mathfrak{S}_{\mu}$ acts as a projector, naturally composing $\mathscr{F}^{D}(G)$ into classes:

$$
\mathscr{F}^{D}(G)=\bigcup_{\mathscr{F} \text { such that } \Im_{\mu}(\mathfrak{F})=\mathscr{F}^{\prime}}\left\{\mathscr{F}^{\prime} \in \mathfrak{F}^{D}(G) \mid \Theta_{\mu}\left(\mathfrak{F}^{\prime}\right)=\mathscr{F}\right\} .
$$

2 Dangerous forests correspond to the skeleton forests of [CR, CPR, R] 
In fact we can characterize $\left\{\mathfrak{F}^{\prime} \in \mathfrak{F}^{D}(G) \mid \mathfrak{S}_{\mu}(\mathfrak{F})=\mathfrak{F}\right\}$ completely in terms of $\mathfrak{H}_{\mu}(\mathfrak{F})=\left\{g \leqq G \mid g\right.$ is connected, divergent, compatible with $\left.\mathfrak{F}, g \in \mathfrak{D}_{\mu}(\mathfrak{F} \cup\{g\})\right\}$.

Lemma 2.3. Let $\mathfrak{F}=\mathfrak{S}_{\mu}(\mathfrak{F})$. Then

(a) $\mathfrak{F} \cup \mathfrak{H}_{\mu}(\mathfrak{F}) \in \mathfrak{F}^{D}(G)$, and

(b) for any $\mathfrak{F}^{\prime} \in \mathfrak{F}^{D}(G)$

$$
\mathfrak{S}_{\mu}(\mathfrak{F})=\mathfrak{F} \Leftrightarrow \mathfrak{F} \cong \mathfrak{F}^{\prime} \cong \mathfrak{F} \cup \mathfrak{H}_{\mu}(\mathfrak{F}) .
$$

This lemma which will be proven later allows us to rewrite

$$
\begin{aligned}
& \mathfrak{R}^{D} G \equiv \sum_{\widetilde{F} \in \widetilde{\Im}^{\mathbb{D}}(G)} \prod_{g \in G}\left(-\mathbb{C}_{g}\right) \sum_{\mu \in \mathfrak{M}} G(\mu)
\end{aligned}
$$

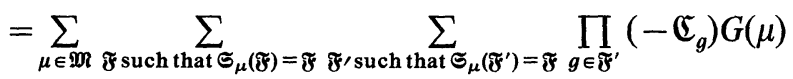

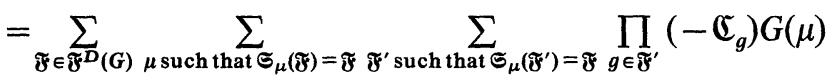

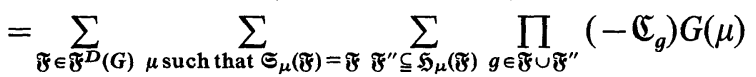

$$
\begin{aligned}
& =\sum_{\mathfrak{F}_{\in} \in \mathfrak{F}^{\mathbb{D}}(\boldsymbol{G})} \sum_{\mu \text { such that } \mathfrak{F}_{\mu}(\mathfrak{F})=\mathfrak{F}} \prod_{g \in \mathfrak{F}}\left(-\mathfrak{C}_{g}\right) \prod_{g \in \mathfrak{F}_{\mu}(\mathfrak{F})}\left(1-\mathfrak{C}_{g}\right) G(\mu) \text {. }
\end{aligned}
$$

Recall that Taylor operators are implicitly ordered so that $\mathfrak{C}_{g}$ always occurs to the left of $\mathfrak{C}_{g^{\prime}}$ when $g \supseteqq g^{\prime}$. In (2.17) we have a "miraculous" reorganization of the "renormalization + momentum space decomposition" process. Thanks to the $\prod_{(\mathfrak{E})}\left(1-\mathfrak{C}_{g}\right)$ we get a renormalization cancellation which renders finite all divergent subgraphs of $G$ that are dangerous from $\mathfrak{F}$ 's point of view. Thanks to the $\prod_{g \in \mathscr{F}}\left(-\mathfrak{C}_{g}\right)\left[\right.$ with $\left.\mathfrak{S}_{\mu}(\mathfrak{F})=\mathfrak{F}\right]$ no other divergent subgraphs are ever encountered (as

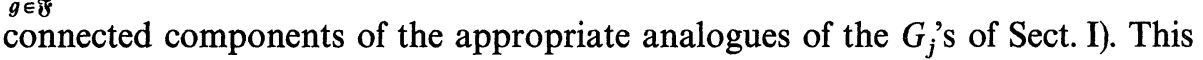
may be seen as follows. We will work inductively, so pick any $g \in \mathfrak{F} \cup \mathfrak{H}_{\mu}(\mathfrak{F}) \cup\{G\}$ and any momentum scale and consider any connected component $h / A_{\mathbb{F}^{\prime} \cup \mathfrak{S}_{\mu}(\mathfrak{\xi})}(h)$ of $\left(g / A_{\Re_{\mathfrak{F}} \cup \mathfrak{F}_{\mu}(\mathfrak{F})}(g)\right)_{j}$. In order for $h$ to be dangerous in the sense that it will fail to deliver any $M^{-\varepsilon}$ 's in the transition from (1.4) to (1.5), it must have $D \geqq 0$. In this case $h$ will be a connected $D \geqq 0$ subgraph that is compatible with $\mathfrak{F} \cup \mathfrak{S}_{\mu}(\mathfrak{F})$ [simply because it is a reexpanded version of a subgraph of $g / A_{\left.\mathscr{F}_{\cup \mathfrak{S}_{\mu}(\mathfrak{F})}(g)\right] \text { and that obeys }}$

$$
\begin{aligned}
i_{h}(\mathfrak{F} \cup\{h\}) & =\min \left\{i_{\ell} \mid \ell \in h / A_{\mathfrak{F} \cup\{h\}}(h)\right\} \\
& =\min \left\{i_{\ell} \mid \ell \in h / A_{\mathfrak{F} \cup \mathfrak{F}_{\mu}(\mathfrak{F}) \cup\{h\}}(h)\right\} \text { by Lemma } 5.2 \\
& \geqq j \\
& >\max \left\{i_{\ell} \mid \ell \in E(h) \cap g\right\} .
\end{aligned}
$$

Now this forces $h=g$ because otherwise

$$
\begin{aligned}
\max \left\{i_{\ell} \mid \ell \in E(h) \cap g\right\} & =\max \left\{i_{\ell} \mid \ell \in E(h) \cap B_{\mathfrak{F} \cup \mathfrak{S}_{\mu}(\mathfrak{F}) \cup(h)}(h)\right\} \\
& =e_{h}(\mathfrak{F} \cup\{h\}) \text { by Lemma } 2.2,
\end{aligned}
$$

and that would place $h \in \mathfrak{H}_{\mu}(\mathfrak{F})$. In the event that $h=g \in \mathfrak{H}_{\mu}(\mathfrak{F})$, renormalization will save the day. In the event that $h=g \in \mathfrak{F}$, which by the way makes it the "useless" 
half of a counterterm that we have been forced to introduce to preserve the locality of the theory, we must still have $i_{h}\left(\mathfrak{F} \cup \mathfrak{H}_{\mu}(\mathfrak{F})\right) \leqq e_{h}\left(\mathfrak{F} \cup \mathfrak{H}_{\mu}(\mathfrak{F})\right)$. This places an upper bound on the possible values of $i_{h}$ preventing divergence.

By hypothesis $(\mathrm{H} 2.5)$ there are at most $K^{\prime \prime L(G)}$ forests in $\mathfrak{F}^{D}$, so it suffices to consider two arbitrary but fixed mutually compatible disjoint forests $\mathfrak{F}, \mathfrak{H} \in \mathfrak{F}^{D}(G)$ and bound

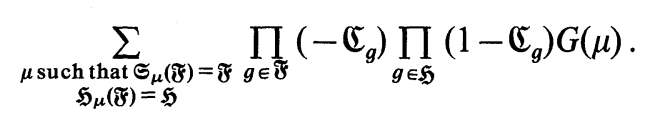

The bound will be by induction on the depth of the forest $\mathfrak{F} \cup \mathfrak{H} \cup\{G\}$. The depth of a forest $\mathscr{F}^{\prime}$ is the size of the largest linear subforest $\left\{g_{1} \subset g_{2} \subset g_{3} \ldots \subset g_{n} \mid g_{i} \in \mathscr{F}^{\prime}\right\}$. This induction can be implemented because given any fixed $g \in \mathfrak{F} \cup \mathfrak{S} \cup\{G\}$, the restrictions of the conditions $\mathfrak{S}_{\mu}(\mathfrak{F})=\mathfrak{F}, \mathfrak{H}_{\mu}(\mathfrak{F})=\mathfrak{H}$ to graphs containing $g$ depend only on momentum assignments to the lines of $G / g$, while the restrictions to graphs contained in or equal to $g$ depend only on the momentum assignments to the lines of $g \cup E(g)$. We will view $g$ as having:

- regular vertices

- generalized vertices. There is one generalized vertex for each

$$
h \in \mathfrak{A}_{\mathfrak{F} \cup \mathfrak{S}}(g)=\left\{h \in \mathfrak{F} \cup \mathfrak{H} \mid h \subset g, h \text { maximal, i.e. } h \subset h^{\prime} \cong g, h^{\prime} \in \mathfrak{F} \cup \mathfrak{H} \Rightarrow h^{\prime}=g\right\} .
$$

The "value" of a generalized vertex $h$ is a distribution which depends not only on the positions $\mathbf{x}$ of the external vertices but also on parameters $\mathbf{e}_{h}=\left\{e_{v} \mid v \in \mathfrak{B}_{E}(h)\right\}$ with $e_{v}$ being thought of as the largest momentum assignment of any external line of $h$ hooked to $v$. The value of the vertex is the obvious analogue of (2.19). It will be denoted $\mathfrak{R}$.

- lines: The set of lines left (i.e. not inside $g$ 's generalized vertices) are simply those of $g / A_{\mathfrak{F} \cup \mathfrak{S}}(g)$. In the event that $g$ is itself a generalized vertex, i.e. in $\mathfrak{F} \cup \mathfrak{H}$, the momentum assignments to these lines must be consistent with the appropriate inequality $i_{g} \leqq e_{g}$ or $i_{g}>e_{g}$, with $e_{g}$ being determined by the parameter values $\mathbf{e}_{g}$. In any event the only connected component $g^{\prime} / A_{\tilde{\mho} \cup \mathfrak{S}}(g)$ of the $\left(g / A_{\mathfrak{F} \cup \mathfrak{S}}(g)\right)_{j}$ 's which may violate $D\left(g^{\prime}\right)<0$ is $g / A_{\mathfrak{F} \cup \mathfrak{S}}(g)$ itself. The momentum assignments to the lines of $g / A_{\mathfrak{g} \cup \mathfrak{S}}(g)$ together with the parameter values $\mathbf{e}_{g}$ determine the parameter values $\mathbf{e}_{h}$ for the generalized vertices of $g$.

We shall prove inductively a bound on $\Re g(\mathbf{x}, \mathbf{e})$ smeared against a test function $f(\mathbf{x})$. The bound is, for $g \in \mathfrak{F} \cup \mathfrak{Y} \cup\{G\}$,

$$
\left|\int \Re g \cdot f d \mathbf{x}\right| \leqq K_{1}^{L(g)} M^{D(g) e_{g}} \lambda_{u(g)}\left(e_{g}\right) \sup _{\mathbf{j} \in T(g)}\left\|\prod_{\substack{v \in \mathfrak{B}_{E}(g) \\ v \neq v_{g}}}\left\{\left(\frac{\nabla x_{v}}{M^{e_{g}}}\right)^{j_{v}} M^{-w j_{v}}\right\} f\right\|_{g}
$$

Here

$$
\lambda_{n}(m)=\sum_{i>m}(i+1)^{n} M^{-\frac{\varepsilon}{2}(i-m)}
$$

[ $\lambda$ gives the accumulated effect of all the $i_{g^{\prime}}$ 's generated by "useless" counterterms (recall (2.7))]

$$
u(g)=\left|\left\{g^{\prime} \in \mathfrak{F} \mid g^{\prime} \cong g\right\}\right| .
$$

$T(g)$ is the set of possible configurations of derivatives that may be applied to $f$. It has the following properties: 
- if $\mathbf{j} \in T(g)$ with $g \in \mathfrak{F}$, then $|\mathbf{j}| \equiv \sum_{v} j_{v} \leqq D(g)$ and $j_{v_{g}}=0$. Hence derivatives generated by $\mathfrak{C}_{h}$ 's with $h \subset g$ cannot get out of $g$.

- if $\mathbf{j} \in T(g)$ with $g \in \mathfrak{H} \cup\{G\}$, then

(i) for each $v \in \mathfrak{B}_{E}(g) 0 \leqq j_{v} \leqq \sum_{\substack{v \in g^{\prime} \in \mathfrak{S} \\ g \geq g^{\prime} \in A_{\mathfrak{F}}(g)}}\left(D\left[g^{\prime}\right]+1\right)+\sum_{\substack{g^{\prime} \in A_{\gamma^{\prime}}(g) \\ v \in g^{\prime}}} D[g]$,

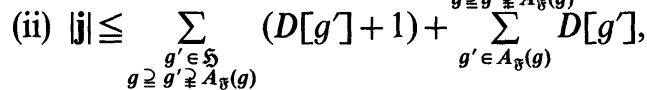

(iii) $j_{v_{g}}=0$.

$v_{g}$ is the fixed vertex of $g$. Its choice will be described later.

$$
w=1 / 2(\bar{D}+1)
$$

The norm $\|\cdot\|_{g}$ is defined as follows. First, for each $h \in \mathfrak{F}, h \cong g$, set equal all variables of $f$ which correspond to the positions of external vertices of $h$. In other words collapse each $h$ to a point. Call the result $(f / \mathfrak{F})\left(\mathbf{x}_{r}\right)$. Then take local suprema in all variables except that containing $v_{g}$,

$$
(\overline{f / \mathfrak{F}})^{e_{g}}\left(\mathbf{x}_{r}\right)=\sup \left\{\left|(f / \mathfrak{F})\left(\mathbf{x}_{r}^{\prime}\right)\right|:\left|x_{v}^{\prime}-x_{v}\right| \leqq\left[\begin{array}{ll}
0, & v=v_{g / \mathfrak{F}} \\
M^{-\left(e_{g}+1\right)}, & v \neq v_{g / \mathfrak{F}}
\end{array}\right\} .\right.
$$

Then multiply by the usual decay factor

$$
g_{e_{g}}\left(\mathbf{x}_{r}\right)=\sup _{\left\{x_{v} \mid v \in \mathfrak{B}_{I}(g / \mathscr{F})\right\}} \prod_{\ell \in g / \mathscr{F}}\left\{e^{-(1-\zeta) M^{e_{0}+1}\left|x_{\ell}-y_{\ell}\right|}\right\} M^{d\left(e_{g}+1\right)\left(V_{E}(g / \mathscr{F})-1\right)}
$$

(the factors of $M$ in $g_{e_{g}}$ are to make it power counting neutral) and integrate

$$
\|f\|_{g}=\left\{\begin{array}{lll}
\int d^{d} x|f(x, x, \ldots, x)| & \text { if } & g \in \mathfrak{F} \\
\int d \mathbf{x}_{r} g_{e_{g}}\left(\mathbf{x}_{r}\right)(\overline{b / \mathfrak{F}})^{e_{g}}\left(\mathbf{x}_{r}\right) & \text { if } & g \in \mathfrak{S} \cup\{G\} .
\end{array}\right.
$$

Applying the inductive bound (2.20) and the facts that $|\mathfrak{F} \cup \mathfrak{H} \cup\{G\}| \leqq L(G)$, $\lambda_{n}(-1) \leqq C_{2}^{n} n$ ! [proven in Lemma (2.4)], $D\left(g^{\prime}\right) \leqq \bar{D}$ [by (2.9)] and

$$
\int d \mathbf{x}_{r} g_{-1}\left(\mathbf{x}_{r}\right)(\overline{f / \mathscr{F}})^{-1}\left(x_{r}\right) \leqq K^{L(G)} \int d \mathbf{x} g_{-1}(\mathbf{x}) \bar{f}^{-1}(\mathbf{x})
$$

(i.e. removing the restriction that some subgraphs be collapsed increases the norm) gives Theorem 2.1.

The heart of this chapter is the proof that the bound (2.20) is preserved by an inductive step. So let $g \in \mathfrak{F} \cup \mathfrak{Y} \cup\{G\}$, and suppose (2.20) is obeyed by all elements of $\mathfrak{A}_{\mathfrak{Z} \cup \mathfrak{S}}(g)$. [The induction would start with $\mathfrak{Q}_{\mathfrak{Z} \cup \mathfrak{S}}(g)=\emptyset$.] We will start by writing explicit formulae only for the case $\mathfrak{A}_{\mathbb{Z} \cup \mathfrak{S}}(g)$ containing a single subgraph $h$. The case $\mathfrak{U}_{\mathfrak{F} \cup \mathfrak{H}}(g)=\emptyset$ is similar but much easier. The general case simply involves taking a tensor product of $h$ 's. In fact in the inductive bound (2.20) we really should have a tensor product of $\Re g$ 's smeared against a single test function $f$. However this would not introduce any new features to the bound. Let $\mathbf{x}(\mathbf{y}, \mathbf{z})$ denote the position

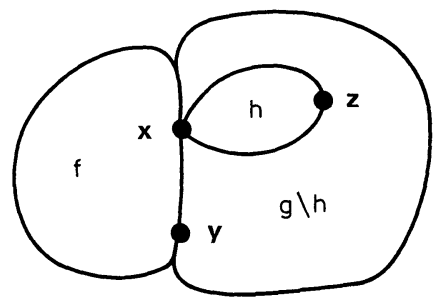


of all vertices common to $f, g$, and $h$ ( $f$ and $g$ only, $h$ and $g$ only) and let $\mathcal{C}_{g}^{\#}$ denote $\mathfrak{C}_{g},\left(1-\mathfrak{C}_{g}\right)$ or 1 as appropriate

$$
\begin{aligned}
& \quad \sum_{\substack{\text { momentum assignments } \\
\text { to } g \backslash h}} \int d \mathbf{x} d \mathbf{y} f(\mathbf{x}, \mathbf{y}) \mathfrak{C}_{g}^{\#}\left[\int d \mathbf{z}(g \backslash h)(\cdot, \cdot, \mathbf{z})(\mathfrak{R} h)(\cdot, \mathbf{z})\right](\mathbf{x}, \mathbf{y}) \\
& =\sum \int d \mathbf{x} d \mathbf{z}(\mathfrak{R} h)(\mathbf{x}, \mathbf{z}) \int d \mathbf{y}\left(\mathbb{C}_{g}^{\# *} f\right)(\mathbf{z}, \mathbf{y})(g \backslash h)(\mathbf{x}, \mathbf{y}, \mathbf{z}) \\
& =\sum \int d \mathbf{x} d \mathbf{z}(\Re h)(\mathbf{x}, \mathbf{z}) f_{0}(\mathbf{x}, \mathbf{z}) .
\end{aligned}
$$

Now apply (2.20) to $\mathfrak{R} h$. The bound on the result is organized into a number of modules:

Module 1. Counting where derivatives can act.

Module 2. Extracting $\left\|\prod_{v}\left\{M^{-w j_{v}^{2}}\left(\frac{\nabla_{x_{v}}}{M^{e_{g}}}\right)^{j_{v}}\right\} f\right\|_{g}$ when $g \in \mathfrak{F}$.

Module 3. Extracting $\left\|\prod_{v}\left\{M^{-w j_{v}^{2}}\left(\frac{\nabla_{x_{v}}}{M^{e_{g}}}\right)^{j_{v}}\right\} f\right\|_{g}$ when $g \in \mathfrak{S} \cup\{G\}$.

Module 4. Extracting power counting factors from renormalization

Module 5. The choice of fixed vertices and the integral over position space.

Module 6. The sum over momentum assignments.

Here we go.

Module 1. Counting where derivatives can act.

Each $\mathbb{C}_{g}^{\#}$ will have up to $D[g]+1$ terms each being a product of up to $D[g]+1$ derivatives each of which can act on any one of $E(g)$ external lines. That's a total of up to

$$
\prod_{g \in \mathfrak{F} \cup \mathfrak{S}}(D[g]+1) E(g)^{D[g]+1} \leqq \operatorname{const}(\bar{D}, \bar{E})^{|\widetilde{8} \cup \mathfrak{S}|}
$$

possibilities, thanks to (H 2.4). Every $g \in \mathfrak{F} \cup \mathfrak{H}$ must contain a line that is not contained in $A_{\mathfrak{Z} \cup \mathfrak{S}}(g),|\mathfrak{F} \cup \mathfrak{H}| \leqq L(g)$, and we can absorb the above constant into $K^{L(G)}$. That's the last counting of this nature that we shall do.

Module 2. Extracting $\left\|\prod_{v}\left\{M^{-w j_{v}^{2}}\left(\frac{\nabla_{x_{v}}}{M^{e_{g}}}\right)^{j_{v}}\right\} f\right\|_{g}$ when $g \in \mathfrak{F}$.

Let's first see what happens to all the derivatives: the old derivatives from $\left\|\left(\frac{\nabla}{M^{e_{h}}}\right)^{\mathbf{j}} f_{0}\right\|_{h}$ and the new derivatives from $\mathfrak{C}_{g}^{*}$. We choose $v_{E}$ of $(2.6)$ to be the fixed vertex $v_{g}$ of $g$ and apply $\mathfrak{C}_{g}^{*}$ to $f$. This produces a sum of terms each a "product" of up to $D[g]$ differential operators $M^{e_{g}}\left(x_{v}-x_{v_{g}}\right) \cdot \frac{\nabla_{x_{v}}}{M^{e_{g}}}$ applied to $f$. The reason for the quotation marks on "product" is that the $\nabla_{x_{v}}$ 's must act directly on $f$ and not on other $\left(x_{v}-x_{v_{g}}\right)$ 's. Furthermore all dependence of $\left(\mathcal{C}_{g}^{*} f\right)$ on all the $x_{v}$ 's other than $x_{v_{g}}$ resides in the $\left(x_{v}-x_{v_{g}}\right)$ 's. Hence as long as we are careful never to differentiate with respect to $x_{v_{g}}$ (that's the content of Module 4) every $\nabla_{x_{v}} / M^{e_{h}}$ from the $\|\cdot\|_{h}$ must act either on $M^{e_{g}}\left(x_{v}-x_{v_{g}}\right)$, yielding $M^{e_{g}-e_{h}}$ or on a propagator of $g \backslash h$. By hypothesis (H 2.1) the effect of applying $n$ such derivatives to a propagator is simply to multiply the conventional estimate $K^{\prime} M^{i_{\ell} \delta(\ell)} e^{-M^{i_{\ell}}\left|x_{\ell}-y_{\ell}\right|}$ by $e^{n^{2-v}}$. (Note that $i_{\ell} \leqq e_{h}$ ). The $e^{n^{2-v}}$ is beaten by the appropriate $M^{-w j_{v}^{2}}$ from inside $\|\cdot\|_{h}$. 
Now we apply $\|\cdot\|_{h}$. If $h \in \mathfrak{F}$ this simply means setting all vertices of $\mathbf{x}$ and $\mathbf{z}$ equal and integrating. In other words just collapse $h$ to a point and treat it like an ordinary vertex. The factor $M^{D(h) e_{h}} \lambda_{u(h)}\left(e_{h}\right)$ should be associated with the vertex and viewed as simply a logarithmic correction $[$ if $u(h) \neq 0]$ to the ordinary powercounting. If $h \in \mathfrak{H}$ we first have to reduce each subgraph of $h$ that is in $\mathfrak{F}$ to a point. Then we have to take some local suprema. Since $x_{v_{g / \mathscr{F}}}$ is the only variable which now appears in $f$ and since either $v_{g}$ is not in $h$, or in the event that it is in $h, v_{g}=v_{h}$ (Module 4 again) it is not involved at all in the local suprema. Hence local suprema are applied only to propagators of $g \backslash h$ that end at vertices of $h$ and to $M^{e_{g}}\left(x_{v}-x_{v_{g}}\right)$ 's having $v \in \mathfrak{B}_{E}(g) \cap \mathfrak{B}_{E}(h)$. In the former case we apply

$$
\left|x_{\ell}^{\prime}-x_{\ell}\right| \leqq M^{-\left(e_{n}+1\right)} e^{-M^{i}\left|x_{\ell}^{\prime}-y_{t}\right|} \leqq e e^{-M^{i} \ell\left|x_{\ell}-y_{\ell}\right|},
$$

since $e_{h} \geqq i_{\ell}$, and in the latter case we use

$$
\sup _{\left|x_{v}^{\prime}-x_{v}\right| \leqq M-\left(e_{n}+1\right)} M^{e}\left|x_{v}^{\prime}-x_{v_{g}}\right| \leqq M^{e_{g}-e_{h}}+M^{e_{g}}\left|x_{v}-x_{v_{g}}\right| .
$$

Hence the only effect of the local suprema is the introduction of additional constants that we merrily absorb into the $K^{L(G)}$ as in Module 1 .

The obvious application of

$$
\int d x_{v_{g}}\left|\varrho\left(x_{v_{g}}\right) \sigma\left(x_{v_{g}}\right)\right| \leqq \sup _{x_{v_{g}}}\left|\varrho\left(x_{v_{g}}\right)\right| \int d x_{v_{g}}\left|\sigma\left(x_{v_{g}}\right)\right|
$$

now leaves us with $\left\|M^{-w \mathbf{j}^{2}}\left(\frac{\nabla}{M^{e_{g}}}\right)^{\mathbf{j}} f\right\|_{g}$ (recall $\mathbf{j}^{2} \leqq$ const) times a graph which consists of $g$ with

(i) each subgraph $h$ in $\mathfrak{A}_{\mathfrak{F} \cup \mathfrak{S}}(g) \cap \mathfrak{F}$ collapsed to a point and endowed with the power counting factor $M^{D(h) e_{n}} \lambda_{u(h)}\left(e_{h}\right)$,

(ii) each subgraph $h$ in $\mathfrak{A}_{\mathfrak{g} \cup \mathfrak{S}}(g) \cap \mathfrak{H}$ replaced by

$$
M^{D(h) e(h)} \lambda_{u(h)}\left(e_{h}\right) h_{e_{h}}\left(\mathbf{x}_{r}\right),
$$

(iii) each line of $g / A_{\mathfrak{\Im} \cup \mathfrak{S}}(g)$ endowed with the usual

$$
M^{i} \delta(\ell) e^{-M_{\ell} i_{\ell}\left|x_{\ell}-y_{\ell}\right|}
$$

(iv) the vertex $v_{g}$ held fixed but all others [consistent with (i), (ii)] integrated over all space,

(v) at most $D[g]$ additional factors of $M^{e_{g}-e_{h}}, M^{e_{g}}\left|x_{v}-x_{v_{g}}\right|$ with $v \in \mathfrak{B}_{E}(g)$.

Module 3. Extracting $\left\|\prod_{v}\left\{M^{-w j_{v}^{2}}\left(\frac{\nabla_{x_{v}}}{M^{\boldsymbol{e}_{g}}}\right)^{j_{v}}\right\} f\right\|_{g}$ when $g \in \mathfrak{S} \cup\{G\}$.

Start by applying to $f_{0}$ in (2.27) the old derivatives $\nabla_{x_{v}} / M^{e_{h}}$ from the inductive bound on $\mathfrak{R} h$. These derivatives can either act on propagators in $g \backslash h$ or on $\left(1-\mathfrak{C}_{g}^{*}\right) f$. In the former case they are treated just as in Module 2 . The inequality $(n+m)^{2} \geqq n^{2}+m^{2}$ may be used to split $M^{-w j_{v}^{2}}$ into two parts: one for transmission forward to the bound on $g$, the other to control the $n$-dependence in our bound on $\nabla^{n} G_{i_{i}}$. In the latter case we use

$$
\left(\frac{\nabla}{M^{e_{h}}}\right)^{\mathbf{j}^{\prime}}\left(1-\mathfrak{C}_{g}^{*}\right) f=\left(\frac{\nabla}{M^{e_{h}}}\right)^{\mathbf{j}^{\prime}} f-\left.\sum_{k=0}^{D[g]} \frac{1}{k !} \frac{d^{k}}{d t^{k}}\left\{t^{\left|\mathbf{j}^{\prime}\right|}\left[\left(\frac{\nabla}{M^{e_{h}}}\right)^{\mathbf{j}^{\prime}} f\right](\mathbf{x}(t))\right\}\right|_{t=0},
$$


which is true because no $\nabla / M^{e_{h}}$ can differentiate the fixed vertex of $g$ which is playing the role of $v_{E}$ in (2.6). If there exists at least one pair of external vertices $v, v^{\prime}$ of $g$ that are separated by at least a distance $\frac{1}{3} M^{-\left(e_{g}+1\right)}$ we need not renormalize $g$ at all, as we shall shortly see. In this case split the right-hand side of (2.31) into the $f$ half and the $\mathfrak{C}_{g}^{*} f$ half. Bound the $f$ half by $\left|\left(\frac{\nabla}{M^{e_{g}}}\right)^{\mathbf{j}^{\prime}} f\right|$, noting that since $g \in\{\mathfrak{G}\} \cup\{G\}, e_{h}>e_{g}$. Treat the $\mathfrak{C}_{g}^{*} f$ half exactly as in Module 2. If, for every pair $v, v^{\prime} \in \mathfrak{B}_{E}(g)$ we have $\left|x_{v}-x_{v^{\prime}}\right| \leqq \frac{1}{3} M^{-\left(e_{g}+1\right)}$ apply Taylor's theorem to the function

$$
f_{1}(t)=|t|^{\left|\mathbf{j}^{\prime}\right|}\left[\left(\frac{\nabla}{M^{e_{n}}}\right)^{j^{\prime}} f\right](\mathbf{x}(t))
$$

to rewrite (2.31) as

$$
\left(\frac{\nabla}{M^{e_{n}}}\right)^{\mathbf{j}^{\prime}}\left(1-\mathfrak{C}_{g}^{*}\right) f=\left.\frac{1}{(D(g)+1) !} \frac{d^{D(g)+1}}{d t^{D(g)+1}}\left\{t^{\left|\mathbf{j}^{\prime}\right|}\left[\left(\frac{\nabla}{M^{e_{n}}}\right)^{\mathbf{j}^{\prime}} f\right](\mathbf{x}(t))\right\}\right|_{t=t^{*}}
$$

for some $0<t^{*}<1$. Evaluating the $d / d t^{\prime}$ 's gives a sum of terms each one of which is of the form (up to irrelevant numerical coefficients and powers of $t^{*}$ )

$$
\left(\frac{M^{e_{g}}}{M^{e_{h}}}\right)^{\left|\mathbf{j}^{\prime}\right|}\left(M^{e_{g}}\left(x_{v}-x_{v_{g}}\right)\right)^{\mathbf{j}^{\prime}}\left[\left(\frac{\nabla}{M^{e_{g}}}\right)^{\mathbf{j}^{\prime}+\mathbf{j}^{\prime \prime}} f\right]\left(\mathbf{x}\left(t^{*}\right)\right)
$$

with $\left|\mathbf{j}^{\prime}\right|+\left|\mathbf{j}^{\prime \prime}\right| \geqq D[g]+1$. These factors of $M^{e_{g}} / M^{e_{h}}$ and $M^{e_{g}}\left(x_{v}-x_{v_{g}}\right)$ are crucial they supply the power counting factors that make renormalization work. Note in addition that the $v^{\text {th }}$ argument of $f$ is evaluated at some unknown point within a distance $(1 / 3) M^{-\left(e_{g}+1\right)}$ of $x_{v}$.

We now update the $M^{-w j_{v}^{2}}$ in the inductive bound. At the beginning of the inductive step we had some $M^{-w \mathbf{j}^{2}}$. Part of this was used up bounding propagators but $M^{-w \mathbf{j}^{\prime 2}}$ was saved [the same $\mathbf{j}^{\prime}$ as in (2.33)]. Now take $\left|\mathbf{j}^{\prime}\right|+\left|\mathbf{j}^{\prime \prime}\right|-D[g]-1$ factors of $M^{e_{g}} / M^{e_{h}}$ from (2.33), recall that $|\mathbf{j}|$ is $\sum_{v} j_{v}$, not $\left(\sum \mathbf{j}_{v}^{2}\right)^{1 / 2}$ and argue

$$
M^{-w \mathbf{j}^{\prime 2}}\left(\frac{M^{e_{g}}}{M^{e_{h}}}\right)^{\left|\mathbf{j}^{\prime}\right|+\left|\mathbf{j}^{\prime \prime}\right|-D[g]-1} \leqq M^{-w \mathbf{j}^{\prime 2}} M^{-\left(\left|\mathbf{j}^{\prime}\right|+\left|\mathbf{j}^{\prime \prime}\right|-D[g]-1\right)}
$$

since $e_{h}>e_{g}$ since $g \in \mathfrak{H} \cup\{G\} \leqq \operatorname{const}(M, \bar{D}) M^{-w\left(\mathbf{j}^{\prime}+\mathbf{j}^{\prime \prime}\right)^{2}}$ since $\left|\mathbf{j}^{\prime \prime}\right| \leqq \bar{D}+1$, $w=1 / 2(\bar{D}+1)$. The constant is no problem. See Module 1 .

Now apply the norm $\|\cdot\|_{h}$ to the result of our calculation of $\left(\nabla / M^{e_{h}}\right)^{\mathbf{j}} f_{0}$. This involves: collapsing some points together [that transmits well since $\left.x_{v}=x_{v^{\prime}} \Rightarrow x_{v}\left(t^{*}\right)=x_{v^{\prime}}\left(t^{*}\right)\right]$; assigning $h$ the power counting factor $M^{D(h) e_{h}} \lambda_{u(h)}\left(e_{h}\right)$; possibly replacing $h$ by $h_{e_{h}}\left(\mathbf{x}_{r}\right)$; taking some local suprema (if $\left.h \in \mathfrak{S}\right)$ and integrating. As in Module 2 applying local suprema to propagators and to $\left(x_{v}-x_{v_{g}}\right)$ 's has no practical effect. Applying local suprema to $f$ merely takes variables whose location is already only known to within an accuracy of $(1 / 3) M^{-\left(e_{g}+1\right)}$ and spreads them out an additional $M^{-\left(e_{h}+1\right)} \leqq(1 / 3) M^{-\left(e_{g}+1\right)}$.

The next step is crucial. It involves exploiting the decay of $g$ to convert the $M^{e_{g}} \mid x_{v}-x_{v_{g}}$ 's, $M^{e_{g}-e_{h}}$ 's and, in the case $\left(1-\mathfrak{C}_{g}^{*}\right) f$ was split up, the separation between a pair of external vertices of $g$ into the power counting factors that make renormalization work. The logically correct time to perform this step is now. But 
to highlight it and to avoid breaking the flow of the current argument we are extracting it as a separate module which can be read a bit later.

Finally we have to separate the test function $\left(\nabla / M^{e_{g}}\right)^{\mathbf{j}^{\prime}+\mathbf{j}^{\prime \prime}} f$ from everything else. We will call everything else the graph $g^{\prime}$. There are really three separate terms. The term in which $\left(1-\mathfrak{C}_{g}^{*}\right) f$ was kept as a unit and the two terms $f$ and $-\mathfrak{C}_{g}^{*} f$ when they were split up. For the two cases $\left(1-\mathfrak{C}_{g}^{*}\right) f$ and $f$ the procedure is the following: First apply (2.10) with all $\eta_{v}$ 's being the characteristic function of a ball of radius $(1 / 3) M^{-\left(e_{g}+1\right)}$ multiplied by the normalizing constant const $M^{d\left(e_{g}+1\right)}$. This gives

$$
\int d \mathbf{x}_{r}\left(m g^{\prime}\right)\left(\mathbf{x}_{r}\right)\left(\overline{\left.\left(\bar{\nabla} / M^{e g}\right)^{\mathbf{j}^{\prime}+\mathbf{j}^{\prime \prime}} f\right)^{g}}\left(\mathbf{x}_{r}\right)\right. \text {. }
$$

Now extract from $\left(m g^{\prime}\right)$ all the normalizing constants above and enough decay factors to build $g_{e_{g}}\left(\mathbf{x}_{r}\right)$, noting that $g^{\prime}$ really does contain all the lines of $g / \mathfrak{F}$ each with a decay factor $\exp \left(-M^{e_{g}+1}\left|x_{\ell}-y_{\ell}\right|\right)$ or better. Call what's left $m g^{\prime \prime}$. Finally apply

$$
\left|\int d \mathbf{x}_{r} \varrho\left(\mathbf{x}_{r}\right) \sigma\left(\mathbf{x}_{r}\right)\right| \leqq \sup \left|\varrho\left(\mathbf{x}_{r}\right)\right| \int d \mathbf{x}_{r} \sigma\left(\mathbf{x}_{r}\right)
$$

to separate $\left(m g^{\prime \prime}\right)$ from $g_{e_{g}}\left(\nabla / M^{e_{g}}\right)^{\mathbf{j}^{\prime}+\mathbf{j}^{\prime \prime}} f$. The result is the desired

$$
\left\|M^{-w \mathbf{j}^{2}}\left(\nabla / M^{e g}\right)^{\mathbf{j}} f\right\|_{g}
$$

times a graph having essentially the structure (2.30). The only significant difference is $(2.30 \mathrm{v})$ is modified. Roughly speaking there are either exactly $D(g)+1$ factors of $M^{e_{g}-e_{h}}, M^{e_{g}} \mid x_{v}-x_{v_{g}}$ lor at least one pair of widely separated external vertices. We will make this precise in Module 4. There are two other irrelevant differences: the decay rates of the lines are down by a constant factor; some of the vertices are integrated only over balls of radius $(1 / 3) M^{e_{g}+1}$.

That leaves us with the "split up term - $\mathfrak{C}_{g}^{*} f$." In it, as happened in Module $2, f$ depends only on $x_{v_{g}}$. In fact we can apply

$$
\int d x_{v_{g}}\left|\varrho\left(x_{v_{g}}\right)\right|\left|\sigma\left(x_{v_{g}}\right)\right| \leqq \sup _{x_{v_{g}}}\left|\varrho\left(x_{v_{g}}\right)\right| \int d x_{v_{g}}\left|\sigma\left(x_{v_{g}}\right)\right|
$$

just as in Module 2. This leaves us with the C-type norm of $M^{-w \mathbf{j}^{2}}\left(\frac{\nabla}{M^{e_{g}}}\right)^{\mathbf{j}} f$ times a graph with the structure (2.30). However in fact the C-type norm in (2.24) is bounded by a constant times the $\mathfrak{S} \cup\{G\}$ type norm in (2.24).

Module 4. Extracting power counting factors from renormalization. Suppose $g \in \mathfrak{H}$. At the time this module is invoked [just before (2.10) is used to separate the test function from the graph in Module 3] we have either

- exactly $D[g]+1$ factors of $M^{e_{g}-e_{h}}, M^{e_{g}}\left|x_{v}-x_{v_{g}}\right|$ in the case that $\left(1-\mathfrak{C}_{g}^{*}\right) f$ was not split up or

- a pair external vertices at least a distance of

$$
(1 / 3) M^{-\left(e_{g}+1\right)}-2 M^{-\left(e_{h}+1\right)} \geqq(1 / 6) M^{-\left(e_{g}+1\right)}
$$

in the case that $\left(1-\mathfrak{C}_{g}^{*}\right) f$ was split up.

Now any pair $v, v^{\prime}$ of external vertices are connected by a sequence of propagators in $g / \mathfrak{F}$. At this point in time each of these propagators is endowed with a decay factor $\exp \left(-M^{i}\left|x_{\ell}-y_{\ell}\right|\right)$ with $i \geqq i_{g}(\mathfrak{F} \cup \mathfrak{H})$. By extracting a very small fraction of each of these factors we can build up

$$
\exp \left\{-\left(\zeta / 10 \bar{E}^{2}\right) M^{i_{g}(\widetilde{\Im} \cup \mathfrak{S})}\left|x_{v}-x_{v^{\prime}}\right|\right\}
$$


while still leaving the original propagators with $\exp \left\{-\left(1-\frac{\zeta}{10}\right) M^{i}\left|x_{\ell}-y_{\ell}\right|\right\}$. In the event that $\left|x_{v}-x_{v^{\prime}}\right| \geqq \frac{1}{6} M^{-\left(e_{g}+1\right)}$, we have

$$
\exp \left\{-\frac{\zeta}{10 \bar{E}^{2}} M^{i_{g}(\mathscr{\mho} \cup \mathfrak{S})}\left|x_{v}-x_{v^{\prime}}\right|\right\} \leqq \text { const }\left(\frac{M^{e_{g}}}{M^{i_{g(\mathfrak{I} \cup \mathfrak{H})}}}\right)^{D[g]+1} .
$$

In the other case (and also in Module 2) we have

$$
\left|x_{v}-x_{v_{g}}\right| \exp \left\{-\frac{\zeta}{10 \bar{E}^{2}} M^{i_{g}(\mathscr{\Im} \cup \mathfrak{S})}\left|x_{v}-x_{v_{g}}\right|\right\} \leqq \text { const } M^{-i_{g}(\mathscr{8} \cup \mathfrak{H})}
$$

this also true, both in Module 2 and Module 3, that $e_{h} \geqq i_{g}(\mathfrak{F} \cup \mathfrak{H})$ simply because $h$ must have an external line that is internal to $g / \mathfrak{F} \cup \mathfrak{H}$. So we are now in a position to replace $(v)$ of $(2.30)$ by

$$
\begin{aligned}
& \text { for } g \in \mathfrak{F} \text { at most } D[g] \text { additional factors of } M^{e_{g}-i_{g}(\mathfrak{F} \cup \mathfrak{H})} \\
& \text { for } g \in \mathfrak{H} \text { exactly } D[g]+1 \text { additional factors of } M^{e_{g}-i_{g}(\mathfrak{F} \cup \mathfrak{H})} \text {. }
\end{aligned}
$$

Module 5. The choice of fixed vertices and the integral over position space. Let $v_{G}$ be any external vertex of $G$ and $\mathfrak{F}^{\prime}$ any forest of connected subgraphs of $G$ that contains $G$. We show how to select from each $g^{\prime} \in \mathfrak{F}^{\prime}$ an external vertex $v_{g^{\prime}}$ in such a way that

(i) $v_{g}$ is the external vertex of $G$ selected.

(ii) for each $g^{\prime} \in \mathfrak{F}^{\prime}$ either $v_{g^{\prime}} \notin A_{\mathscr{F}^{\prime}}\left(g^{\prime}\right)$ or for all $h^{\prime} \in \mathfrak{F}^{\prime}$ with $v_{g^{\prime}} \in h^{\prime} \subset g^{\prime}$ we have $v_{h^{\prime}}=v_{g^{\prime}}$.

Work by induction from large subgraphs to small ones using the partial ordering of $\mathfrak{F}^{\prime}$ by inclusion. We pick $v_{G}$ for $G$ to start. Then:

$$
\begin{aligned}
& \text { if } v_{\boldsymbol{B}_{\mathscr{F}^{\prime}}\left(h^{\prime}\right)} \in h^{\prime} \text { then set } v_{h^{\prime}}=v_{\boldsymbol{B}_{\mathbb{F}^{\prime}}\left(h^{\prime}\right)} \\
& \text { if } v_{\boldsymbol{B}_{\mathscr{\gamma}^{\prime}}\left(h^{\prime}\right)} \notin h^{\prime} \text { then choose } v_{h} \text { at random from amongst } \mathfrak{B}_{E}\left(h^{\prime}\right) .
\end{aligned}
$$

Applying this rule to $\mathfrak{F}^{\prime}=\mathfrak{F} \cup \mathfrak{S}$ builds the $v_{g}$ 's which appear in (2.20), and they satisfy (i) and (ii).

Now the current situation as far as $x$ dependence is concerned is the following. [See (2.30)]

- Each subgraph $\mathfrak{U}_{\mathfrak{F} \cup \mathfrak{H}}(g) \cap \mathfrak{F}$ has been collapsed to a point. This point is integrated over all space.

- Each subgraph $h$ in $\mathfrak{A}_{\mathfrak{F} \cup \mathfrak{H}}(g) \cap \mathfrak{H}$ has been replaced by $h_{e_{h}}\left(\mathbf{x}_{r}\right)$. By (2.25), this means that $h$ has been replaced by $h / \mathfrak{F}$ (i.e. subgraphs of $h$ that are in $\mathfrak{F}$ have been collapsed), that internal vertices of $h / \mathfrak{F}$ are not integrated over, that external vertices of $h / \mathfrak{F}$ are integrated and that propagators in $h / \mathfrak{F}$ have decay rate const $M^{e_{h}+1}$;

- Lines in $g / \mathfrak{F} \cup \mathfrak{H}$ have decay rates const $M^{i_{\ell}}$ as usual.

- One vertex $v_{g}$ is held fixed but all others not in $A_{\mathscr{\mho} \cup \mathfrak{H}}(g)$ are integrated over all space.

The only impediment to our immediately applying the procedure of Sect. I is the fact that vertices internal to subgraphs of $\mathfrak{U}_{\mathfrak{Z} \cup \mathfrak{S}}(g) \cap \mathfrak{S}$ are not integrated over. This problem is avoided by simply getting rid of them: deleting internal vertices and some internal lines, using triangular inequality, we bound each such $h$ by a tree 
connecting the external vertices of $h$. The lines of this tree have decay const $M^{e_{h}+1}$. We now have a conventional graph $h$ [namely $g / \mathfrak{F}$ but with each subgraph in $\mathfrak{U}_{\mathfrak{F} \cup \mathfrak{S}}(g) \cap \mathfrak{S}$ replaced by a tree connecting its external vertices] each of whose vertices, with the exception of one fixed vertex $v_{g}$, are to be integrated over all space. Now integrate over position space as in Sect. I (or [FMRS1,2]). This gives, for each vertex $v$ in $g^{\prime}$, a factor const $M^{-d i_{v}}$, where $i_{v}$ is the momentum scale in which $v$ was integrated. The constants just go to updating the $K_{1}^{L(g)}$ of the inductive bound (2.20). Hence our bound on $\left|\int \Re g \cdot f d \mathbf{x}\right|$ now consists of

$$
K_{1}^{L(g)} \sup _{\mathbf{j} \in T(g)}\left\|\prod_{\substack{v \in \mathfrak{B}_{E}(g) \\ v \neq v_{g}}}\left\{M^{-w j_{v}^{2}}\left(\frac{\nabla x_{v}}{M^{e_{g}}}\right)^{j_{v}}\right\} f\right\|_{g}
$$

times the sum over all possible momenta assignments of a bunch of momentum factors:

$-M^{-d i v}$ for each $v \neq v_{g}$ of $g^{\prime}$

- $M^{i, \delta(\ell)}$ for each line of $g / A_{\mathfrak{F} \cup \mathfrak{H}}(g)$ from (2.30)(iii)

$-M^{D(h) e_{h}} \lambda_{u(h)}\left(e_{h}\right)$ for each $h \in \mathfrak{U}_{\mathfrak{F} \cup \mathfrak{S}}(g)$ from (2.30) (i), (ii)

$-M^{d\left(e_{h}+1\right)\left(V_{E}(h / \mathscr{Z})-1\right)}$ for each $h \in \mathfrak{A}_{\mathfrak{F} \cup \mathfrak{H}}(g) \cap \mathfrak{H}$ from (2.30) (ii), (2.25)

$-M^{\left[e_{g}-i_{g}(\mathfrak{F} \cup \mathfrak{S})\right] D_{R}}$ with $D_{R}=D[g]+1$ if $g \in \mathfrak{H}$ and $0 \leqq D_{R} \leqq D[g]$ if $g \in \mathfrak{F}$ from (2.36)(v).

Before moving onto the final module we simply organize these factors a little better. This organization will involve the assignment of factors to the connected components $(g / \mathfrak{F} \cup \mathfrak{H})_{j}^{k}$ of

$$
(g / \mathfrak{F} \cup \mathfrak{H})_{j}=\left\{l \in g / \mathfrak{F} \cup \mathfrak{H} \mid i_{\ell} \geqq j\right\} .
$$

Start by writing

$$
M^{-d i_{v}}=M^{d} \prod_{i=0}^{i_{v}} M^{-d}
$$

and similar decompositions for $\left[M^{\delta(\ell)}\right]^{i_{\ell}},\left[M^{D(h)}\right]^{e_{h}}$ and $\left[M^{d\left(V_{E}(h / F)-1\right)}\right]^{e_{h}+1}$. Now the factors left over in front of the product signs combine to give $M$ to the power

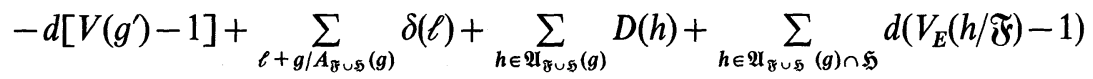

$$
\begin{aligned}
& =-d(V(g / \mathfrak{F} \cup \mathfrak{H})-1)+\sum_{\ell \in g / \mathfrak{A}_{\mathfrak{F} \cup \mathfrak{S}}(g)} \delta(\ell)+\sum_{h \in \mathfrak{A}_{\mathfrak{F} \cup \mathfrak{S}}(g)} D(h) \\
& =D(g / \mathfrak{F} \cup \mathfrak{G})+\sum_{h \in \mathfrak{A}_{\mathfrak{\xi} \cup \mathfrak{I}}(g)} D(h) \\
& =D(g) \text {. }
\end{aligned}
$$

Since $D(g) \leqq \bar{D}$ this $M^{D(g)}$ can be absorbed into the $K^{L(g)}$ as in Module 1. Next consider any connected component $g^{\prime \prime}$ of

$$
g_{j}^{\prime}=\left\{\ell \in g^{\prime} \mid \ell \text { had decay rate } \geqq \text { const } M^{j}\right\} .
$$

Each vertex but one of this component has a factor of $M^{-d}$. In particular if a tree replacing some $h \in \mathfrak{A}_{\mathfrak{Z} \cup \mathfrak{S}}(g) \cap \mathfrak{H}$ appears in $g^{\prime \prime}$ (and it will appear either in whole or not at all) the vertices of the tree thus have $M^{-d V_{E}(h / \mathfrak{F})}$. This combines with an $M^{d\left[V_{E}(h / \mathscr{F})-1\right]}$ from the decomposition of $\left(M^{d\left[V_{E}(h / \mathscr{F})-1\right]}\right)^{e_{h}+1}$ to give just plain old

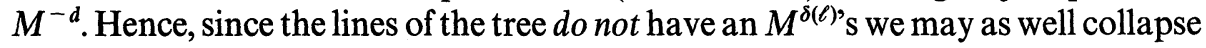


the tree and view it as being a single vertex. After this collapsing the lines of $g_{j}^{\prime}$ are exactly the lines of $(g / \mathfrak{F} \cup \mathfrak{H})_{j}$ and each has an $M^{\delta(\ell)}$ and the vertices consist of the vertices of $(g / \mathfrak{F} \cup \mathfrak{H})_{j}$ plus possibly some isolated vertices. These isolated vertices arise when some $h \in \mathfrak{A}_{\mathfrak{F} \cup \mathfrak{H}}(g) \cap \mathfrak{H}$ has $i_{\ell}<j$ but every line in $E(h) \cap g$ has $i_{\ell}<j$. This vertex has the factor one so we just throw it away. Hence the $M^{-d k_{v}}, M^{i \ell \delta(\ell)}$, $M^{d\left(e_{h}+1\right)\left(V_{E}(h / \mathscr{F})-1\right)}$ have resulted in

$$
\prod_{j, k} M^{D\left(\left(g / \mathfrak{F} \cup \mathfrak{S}_{j}^{(k)}\right)\right.}
$$

Now we look after the factors resulting from the decomposition of the $\left[M^{D(h)}\right]^{e_{h}}$ 's. Since

$$
e_{h}=\max \left\{i_{\ell} \mid \ell \in E(h) \cap g\right\},
$$

$h$ is a vertex of $(g / \mathfrak{F} \cup \mathfrak{S})_{j}$ for exactly $e_{h}+1$ values of $j$ and it is natural to put $M^{D(h) e_{h}}$ into (2.39).

Module 6. The sum over momentum assignments.

We are left with the problem of controlling the sum over allowed momentum assignments of

$$
M^{\left(e_{g}-i_{g}(\mathfrak{F} \cup \mathfrak{H})\right) D_{R}} \prod_{h \in \mathfrak{Q}\{\cup \mathfrak{S}(g)} \lambda_{u(h)}\left(e_{h}\right) \prod_{j, k} M^{\bar{D}\left((g / \Im \cup \mathfrak{H})_{j}^{k}\right)},
$$

where

$$
\begin{aligned}
\bar{D}\left((g / \mathfrak{F} \cup \mathfrak{H})_{j}^{k}\right) & =D\left((g / \mathfrak{F} \cup \mathfrak{H})_{j}^{k}\right)+\sum_{\begin{array}{c}
h \in \mathfrak{A}_{\mathcal{F} \cup \mathfrak{S}}(g) \\
\text { such that } h \text { is a vertex } \\
\text { of }(g / \mathfrak{F} \cup \mathfrak{S})_{j}^{k}
\end{array}} D(h) \\
& =D\left(g_{j}^{k}\right) .
\end{aligned}
$$

Here $g_{j}^{k}$ denotes the subgraph of $g$ consisting of $(g / \mathfrak{F} \cup \mathfrak{H})_{j}^{k}$ with all the collapsed subgraphs reexpanded. Now by $(2.18),(2.19) D\left(g_{j}^{k}\right)$ can be non-negative only for $g_{j}^{k}=g$. For all other $g_{j}^{k}$, ,

$$
D\left(g_{j}^{k}\right) \leqq-2 \varepsilon E\left(g_{j}^{k}\right)=-2 \varepsilon E\left((g / \mathfrak{F} \cup \mathfrak{H})_{j}^{k}\right) .
$$

Hence we are almost in the position of (1.5). The only problem is that we can't harvest any decay for $j \leqq i_{g}(\mathfrak{F} \cup \mathfrak{S})$ :

$$
\prod_{j, k} M^{\bar{D}\left((g / \mathfrak{F} \cup \mathfrak{H})_{j}^{k}\right)} \leqq M^{D(g)\left[i_{g}(\mathfrak{F} \cup \mathfrak{H})+1\right]} \prod_{\substack{j, k \\ j>i_{g}(\mathfrak{F} \cup \mathfrak{H})}} M^{-2 \varepsilon E\left((g / \mathfrak{\Im} \cup \mathfrak{H})_{j}^{k}\right)} .
$$

So we rewrite the sum over all allowed momentum assignments as an iterated sum $\sum_{i_{g}} \sum_{\mu \mid i_{g}}$ with the inside sum being over all allowed momentum assignments having

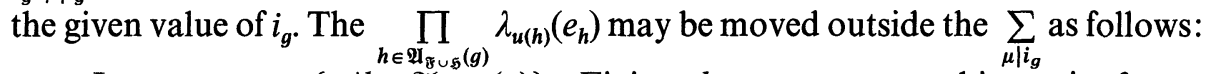
- Let $e_{h_{m}}=\max \left\{e_{\ell} \mid h \in \mathfrak{Q}_{\mathfrak{F} \cup \mathfrak{S}}(g)\right\}$. Fixing $h_{m}$ costs a combinatoric factor $\left|\mathfrak{A}_{\mathfrak{\Im} \cup \mathfrak{S}}(g)\right|$ that may be absorbed in the $K^{L(g)}$ as in Module 1.

- Apply

$$
\begin{aligned}
\prod_{h} \lambda_{u(h)}\left(e_{h}\right) & \leqq \prod_{h} \lambda_{u(h)}\left(e_{h_{m}}\right) \quad(\text { Lemma } 2.4(\mathrm{c})) \\
& \leqq \lambda_{\Sigma u(h)}\left(e_{h_{m}}\right) \quad(\text { Lemma } 2.4(\mathrm{f}))
\end{aligned}
$$


- Extract part of the decay in momentum space that exists between the line of scale $i_{g}$ and the line of $E\left(h_{m}\right) \cap g$ that has scale $e_{h_{m}}$ (again the combinatoric factors involved are no problem) to get $M^{-\frac{3}{4} \varepsilon\left(e_{h_{m}}-i_{g}\right)}$ and apply

$$
\exp \left(-\frac{\varepsilon}{2}\left(e_{h_{m}}-i_{g}\right)\right) \lambda_{\Sigma u(h)}\left(e_{h_{m}}\right) \leqq \lambda_{\Sigma u(h)}\left(i_{g}\right) . \quad(\text { Lemma 2.4(e)) }
$$

That leaves $\prod_{\substack{j, k \\ j>i_{g}(\xi \cup \mathfrak{S})}} M^{-2 \varepsilon E\left[(g / \Im \cup \mathfrak{S})_{j}^{k}\right]}$ inside the sum $\sum_{\mu \mid i_{g}}$. This sum may now be done exactly as in Sect. I and is bounded by $K^{\prime L(g / \Im \cup \mathfrak{F})}$ which may be used to update the $K^{L(g)}$ in the inductive bound (2.20). Our sole remaining task is to sum

$$
\sum_{i_{g}} M^{\left[e_{g}-i_{g}\right] D_{R}} \lambda_{\Sigma u(h)}\left(i_{g}\right) M^{D(g)\left[i_{g}+1\right]} .
$$

If $g \in \mathfrak{F}$ we have

$$
\begin{aligned}
& \sum_{i_{g} \leqq e_{g}} M^{\left[e_{g}-i_{g}\right] D_{R}} \lambda_{\Sigma u(h)}\left(i_{g}\right) M^{D(g)\left[i_{g}+1\right]} \\
& \quad \leqq M^{\bar{D}} M^{D(g) e_{g}} \sum_{i_{g} \leqq e_{g}} \lambda_{\Sigma u(h)}\left(i_{g}\right) \text { since } D_{R} \leqq D(g) \\
& \leqq M^{\bar{D}} M^{D(g) e_{g}}\left(e_{g}+1\right) \lambda_{\Sigma u(h)}\left(e_{g}\right) \text { by Lemma }(2.4 \mathrm{c}) \\
& \quad \leqq \operatorname{const} M^{D(g) e_{g}} \lambda_{\Sigma u(h)+1}\left(e_{g}\right) \text { by Lemma (2.4d) }
\end{aligned}
$$

and this is what we want since $\sum_{h \in \mathfrak{H}_{\mathbb{Z} \cup \mathfrak{S}}(g)} u(h)+1=u(g)$ for $g \in \mathfrak{F}$. If $g \in \mathfrak{S}$ we have

$$
\begin{aligned}
& \sum_{i_{g}>e_{g}} M^{\left[e_{g}-i_{g}\right](D[g]+1)} \lambda_{\Sigma u(h)}\left(i_{g}\right) M^{D(g)\left(i_{g}+1\right)} \\
& \quad \leqq M^{D(g)} M^{D(g) e_{g}} \sum_{i_{g}>e_{g}} M^{\varepsilon\left(e_{g}-i_{g}\right)} \lambda_{\Sigma u(h)}\left(i_{g}\right) \text { since } D[g]+1 \geqq D(g)+\varepsilon \\
& \quad \leqq \text { const } M^{D(g) e_{g}} \lambda_{\Sigma u(h)}\left(e_{g}\right) \text { by Lemma }(2.4 \mathrm{e}) .
\end{aligned}
$$

This is again exactly what we want since again

$$
\sum_{h \in \mathcal{Q} \mathcal{I}_{\mathcal{g}}(g)} u(h)=u(g) \quad \text { for } \quad g \in \mathfrak{F} .
$$

It remains only to prove some by-passed lemmas:

Lemma 2.2. For any $\mathfrak{F} \in \mathfrak{F}^{D}(G)$, assignment of momenta $\mu$ and any connected $g$, compatible with $\mathfrak{F}$

$$
\begin{gathered}
\min \left\{i_{\ell} \mid \ell \in g \backslash A_{\mathfrak{S}_{\mu}(\mathfrak{F}) \cup\{g\}}(g)\right\}=\min \left\{i_{\ell} \mid \ell \in g \backslash A_{\mathfrak{F} \cup\{g\}}(g)\right\}, \\
\max \left\{i_{\ell} \mid \ell \in E(g) \cap B_{\mathfrak{S}_{\mu}(\mathfrak{F}) \cup\{g\}}(g)\right\}=\max \left\{i_{\ell} \mid \ell \in g \cap B_{\widetilde{F} \cup\{g\}}(g)\right\} .
\end{gathered}
$$

Proof. (a) Suppose $A_{\mathcal{\subseteq}_{\mu}(\mathfrak{F}) \cup\{g\}}(g) \varsubsetneqq A_{\mathscr{F}}(g)$ and let $\ell_{0}$ be any line in $A_{\mathscr{F}}(g) \backslash A_{\mathfrak{S}_{\mu}(\mathfrak{F}) \cup\{g\}}(g)$. Let

$$
\ell_{0} \ni d_{0} \subset d_{1} \subset \ldots \subset d_{n} \subset g
$$

be the set of all dangerous elements of $\mathfrak{F}$ containing $\ell_{0}$ and contained in $g$. Then for each $k=0, \ldots, n$ there must exist a line $\ell_{k+1}$ which is an external line of $d_{k}$ and is contained in $d_{k+1}(g$ if $k=n)$. Since $d_{k}$ is dangerous and there aren't any safe sets between $\ell_{0}$ and $g, i_{\ell_{k}}>i_{\ell_{k+1}}$. Hence $i_{\ell_{0}}>i_{\ell_{n+1}}$ with $\ell_{n+1} \in g \backslash A_{\mathfrak{g} \cup\{g\}}$. Hence $\ell_{0}$ cannot provide the minimum in $g \backslash A_{\mathcal{E}_{\mu(\xi)}(g)}(g)$. 
(b) Suppose $E(g) \cap B_{\mathfrak{G}_{\mu}(\mathfrak{F}) \cup[g\}}(g) \nsupseteq E(g) \cap B_{\widetilde{g} \cup\{g\}}(g)$ and let $\ell_{0}$ be any line in the difference. Let $\ell_{1}$ be any line in $E(g) \cap B_{\widetilde{\gamma} \cup\{g\}}(g)$. Let $d$ be the largest set of $\mathfrak{F}$ obeying

$$
g \subset d \subset B_{\mathfrak{\Im}_{\mu}(\mathfrak{F}) \cup\{g\}}, \quad \ell_{0} \in E(d)
$$

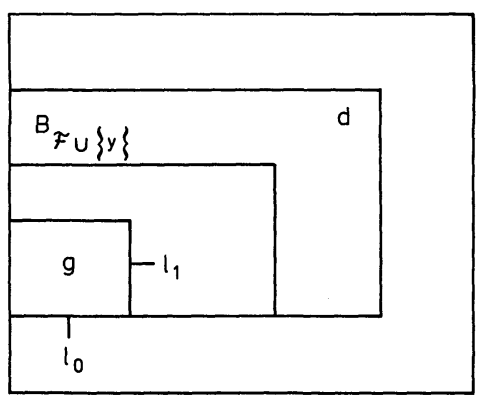

$$
B_{\mathscr{\omega}_{\mu}(\mathfrak{)})}
$$

For example $B_{\mathfrak{F}}(g)$ obeys (2.43). Now $d$ must be dangerous for $\mathfrak{F}$ so using the result of (a) part

$$
i_{\ell_{0}} \leqq e_{d}(\mathfrak{F})<i_{d}(\mathfrak{F})=i_{d}\left(\mathfrak{S}_{\mu}(\mathfrak{F}) \cup\{d\}\right) \leqq i_{\ell_{1}} .
$$

Hence $\ell_{0}$ cannot provide the minimum in $E(g) \cap B_{\widetilde{E}_{\mu}(\Re) \cup\{g\}}(g)$.

Lemma 2.3. Let $\mathfrak{F}=\Im_{\mu}(\mathfrak{F})$. Then

(a) $\mathfrak{F} \cup \mathfrak{H}_{\mu}(\mathfrak{F}) \in \mathfrak{F}^{D}(G)$ and

(b) for any $\mathscr{F}^{\prime} \in \mathscr{F}^{D}(G)$

$$
\mathfrak{S}_{\mu}(\mathfrak{F})=\mathfrak{F} \Leftrightarrow \mathfrak{F} \cong \mathfrak{F}^{\prime} \cong \mathfrak{F} \cup \mathfrak{G}_{\mu}(\mathfrak{F}) .
$$

Proof. (a) We need only show that no two $g, g^{\prime} \in \mathfrak{H}_{\mu}(\mathfrak{F})$ can overlap. If they did $g \backslash g^{\prime}$ and $g \backslash g$ would have to

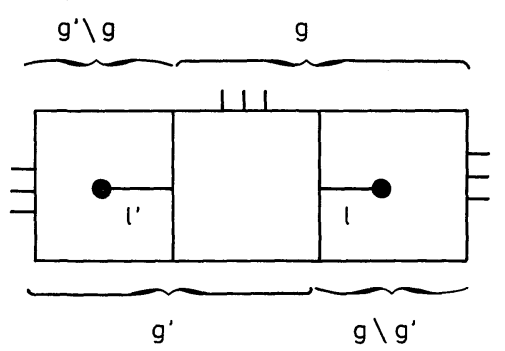

be nonempty and $g$ and $g^{\prime}$ would have to have at least a vertex in common. But since $g$ is connected $g \backslash g^{\prime}$ would then have to contain a line $\ell$ which was an internal line for $g$ and an external line for $g^{\prime}$. Furthermore $\ell$ cannot be in $A_{\mathfrak{F} \cup\{g\}}(g)$ by the compatibility of $g^{\prime}$ with $\mathfrak{F}$ and must be in $B_{\mathscr{\gamma} \cup\left\{g^{\prime}\right\}}\left(g^{\prime}\right)$ by the compatibility of $g$ with F. Similarly $g \backslash g$ would have to contain a line $\ell^{\prime}$ with $\ell^{\prime} \in g \backslash A_{\tilde{g} \cup\left\{g^{\prime}\right\}}\left(g^{\prime}\right)$, $\ell^{\prime} \in E(g) \cap B_{\mathfrak{z} \cup\{g\}}(g)$. But this now forces

and

$$
i_{\ell} \geqq i_{g}(\mathfrak{F} \cup\{g\})>e_{g}(\mathfrak{F} \cup\{g\}) \geqq i_{\ell^{\prime}}
$$

$$
i_{\ell^{\prime}} \geqq i_{g^{\prime}}\left(\mathfrak{F} \cup\left\{g^{\prime}\right\}\right)>e_{g^{\prime}}\left(\mathfrak{F} \cup\left\{g^{\prime}\right\}\right) \geqq i_{\ell} .
$$


That's a contradiction.

(b) Firstly note that if $\mathfrak{F}_{1}, \mathfrak{F}_{2} \in \mathfrak{F}^{D}(G)$ and $\mathfrak{F}_{1} \subseteq \mathfrak{F}_{2}$ then by (2.13):

$$
g \in \mathfrak{D}_{\mu}\left(\mathfrak{F}_{1}\right) \Rightarrow g \in \mathfrak{D}_{\mu}\left(\mathfrak{F}_{2}\right) \text {. }
$$

Hence if $g \in \mathfrak{F}^{\prime}-\mathfrak{F}=\mathfrak{F}^{\prime} \cap \mathfrak{H}_{\mu}(\mathfrak{F})$, then $g \in \mathfrak{D}_{\mu}(\mathfrak{F} \cup\{g\}) \subset \mathfrak{D}_{\mu}\left(\mathfrak{F}^{\prime}\right)$. So $\mathfrak{S}_{\mu}(\mathfrak{F}) \cong \mathfrak{F}$. In order to have $\mathfrak{S}_{\mu}(\mathfrak{F}) \subseteq \mathfrak{F}$, there must be a $g \in \mathfrak{F}$ with

$$
i_{g}\left(\mathfrak{F}^{\prime}\right)>e_{g}\left(\mathfrak{F}^{\prime}\right)=e_{g}\left(\mathfrak{S}_{\mu}\left(\mathfrak{F}^{\prime}\right)\right) \geqq e_{g}(\mathfrak{F}) \geqq i_{g}(\mathfrak{F})=i_{\ell_{0}},
$$

since $\mathfrak{F}=\mathfrak{S}_{\mu}(\mathfrak{F})$. But then $\ell_{0}$ must be in some $h \in \mathfrak{H}_{\mu}(\mathfrak{F})$ with $h \subset g$ and must not be in $A_{\mathfrak{g}}(g)$ and so:

$$
i_{\ell_{0}}=\min \left\{i_{\ell} \mid \ell \in h \backslash A_{\mathscr{F}}(g)=h-A_{\mathscr{J} \cup\{h\}}(h)\right\}>\max \left\{i_{\ell} \mid \ell \in E(h) \cap B_{\mathscr{F} \cup\{h\}}(h)\right\} .
$$

This is impossible because $E(h)$ must contain a line that is internal to $B_{\mathscr{Z} \cup\{h\}}(h) \subseteq g$ [or else $B_{\mathscr{F} \cup\{h\}}(h)$ is not connected] and this line cannot be in $A_{\mathscr{F}}(g)$ [since $h$ is compatible with $\mathfrak{F}$ and is not contained in $A_{\mathfrak{F}}(g)$ ].

$\Rightarrow$ Assume $\mathfrak{S}_{\mu}\left(\mathfrak{F}^{\prime}\right)=\mathfrak{F}$. It is obvious that $\mathfrak{F} \cong \mathfrak{F}^{\prime}$. Furthermore

$$
g \in \mathfrak{F}^{\prime}-\mathfrak{F} \Rightarrow g \in \mathfrak{D}_{\mu}\left(\mathfrak{F}^{\prime}\right) \Rightarrow g \in \mathfrak{D}_{\mu}\left(\mathfrak{S}_{\mu}\left(\mathfrak{F}^{\prime}\right) \cup\{g\}\right.
$$

by (2.44).

Lemma 2.4. There exist constants $c_{i}(\varepsilon)$ such that for all $n>0$
(a) $c_{1}^{n} n ! \leqq \lambda_{n}(-1) \leqq c_{2}^{n} n$ !
(b) $c_{1}^{n} n !+(m+1)^{n} \leqq \lambda_{n}(m) \leqq c_{3}^{n}\left(n !+(m+1)^{n}\right)$ for $m \geqq-1$,
(c) $\lambda_{n}(m) \leqq \lambda_{n}\left(m^{\prime}\right)$ for $m^{\prime} \geqq m$,
(d) $(m+1)^{n} \lambda_{n}(m)=\lambda_{n+n^{\prime}}(m)$,
(e) $\sum_{m^{\prime}>m} M^{-\frac{3}{4} \varepsilon\left(m^{\prime}-m\right)} \lambda_{n}\left(m^{\prime}\right) \leqq \lambda_{n}(m)$ if $M$ is large enough,
(f) $\prod_{p=1}^{k} \lambda_{n_{p}}(m) \leqq \lambda_{\Sigma n_{p}}(m)$ if $M$ is large enough.

Proof. Recall that $\lambda_{n}(m)=\sum_{i>m}(i+1)^{n} M^{-\frac{\varepsilon}{2}(i-m)}=\sum_{i=1}^{\infty}(i+m+1)^{n} M^{-\frac{\varepsilon}{2} i}$

(a)

$$
\begin{gathered}
\sum_{i=1}^{\infty} i^{n} M^{-\frac{\varepsilon}{2} i} \geqq n^{n} M^{-\frac{\varepsilon}{2} n} \geqq n ! c_{1}^{n}, \\
\sum_{i=1}^{\infty} i^{n} M^{-\frac{\varepsilon}{i} i}=\frac{n !}{\alpha^{n}} \sum_{i=1}^{\infty} \frac{1}{n !}(\alpha i)^{n} M^{-\frac{\varepsilon}{2} i} \leqq \frac{n !}{\alpha^{n}} \sum_{i=1}^{\infty} e^{\alpha i} M^{-\frac{\varepsilon}{2} i} \leqq c \frac{n !}{\alpha^{n}}
\end{gathered}
$$

for $\alpha$ small enough.

(b) $\quad \sum_{i=1}^{\infty}(i+m+1)^{n} M^{-\frac{\varepsilon}{2} i}=\sum_{k=0}^{\infty} \sum_{i=1}^{\infty} \frac{n !}{k !(n-k) !}(m+1)^{k} i^{n-k} M^{-\frac{\varepsilon}{2} i}$.

By (a) part this is bounded above and below (for different $c$ 's) by

$$
\sum_{k=0}^{n} \frac{n !}{k !(n-k) !}(m+1)^{k} c^{n-k}(n-k) !
$$

The lower bound follows by taking the $k=0$ and $k=n$ terms. The upper bound follows by $(n-k) !(m+1)^{k}$. (c) and (d) are obvious. 
(e)

$$
\begin{gathered}
\sum_{m^{\prime}>m} M^{-\frac{3}{4} \varepsilon\left(m^{\prime}-m\right)} \lambda_{n}\left(m^{\prime}\right)=\sum_{i>m^{\prime}>m}(i+1)^{n} M^{-\frac{\varepsilon}{2}\left(i-m^{\prime}\right)} M^{-\frac{3}{4} \varepsilon\left(m^{\prime}-m\right)} \\
\leqq \sum_{i>m ; m^{\prime}>m}(i+1)^{n} M^{-\frac{\varepsilon}{2}(i-m)} M^{-\frac{\varepsilon}{4}\left(m^{\prime}-m\right)} \leqq \lambda_{n}(m)
\end{gathered}
$$

if $M$ is large enough.

(f) $\prod_{p=1}^{k}\left(\sum_{i_{p}=1}^{\infty}\left(i_{p}+m+1\right)^{n_{p}} M^{-\frac{\varepsilon}{2} i_{p}}\right) \leqq \sum_{\left\{i_{p}\right\}}\left(m+1+\max _{p} i_{p}\right)^{\Sigma n_{p}} M^{-\frac{\varepsilon}{2} \Sigma i_{p}}$

$$
\begin{aligned}
& \leqq k \sum_{i_{1}=1}^{\infty} \sum_{i_{2} \ldots i_{k} \leqq i_{1}}\left(i_{1}+m+1\right)^{\Sigma n_{p}} M^{-\Sigma \frac{\varepsilon}{2} i_{p}} \\
& \leqq k \lambda_{\Sigma n_{p}}(m)\left(\sum_{i=1}^{\infty} M^{-\frac{\varepsilon}{2} i}\right)^{k-1} \leqq \lambda_{\Sigma n_{p}}(m)
\end{aligned}
$$

provided $M$ is large enough.

\section{Renormalized Graphs: Bounds for $\phi_{4}^{4}$-like Models}

There are two sources of slackness in the $S(G)$ dependence of the bound of Theorem 2.1 at least as far as it applies to models like $\phi_{4}^{4}$. They arise because $\mathfrak{F}^{D}(G)$ is unnecessarily large.

(S1) We have introduced counterterms for 1PR subgraphs even though it is wellknown that these will in fact be finite.

(S2) We have introduced counterterms for non-closed subgraphs. Hence if, for example, $G=\longleftrightarrow$, we introduce counterterms for both $\longrightarrow$ and $\circlearrowright$. This is silly. $x \circlearrowright y$ is a function of only one variable $x-y$. Any non-integrable singularity in $x-y$ can clearly be removed with a single Taylor operation.

These two sources of slackness are removed simply by cutting down $\mathfrak{F}^{D}(G)$ to $\mathfrak{F}^{c}(G)$, the set of closed, proper, divergent forests of $G$ :

$$
\mathfrak{R}^{c} G=\sum_{\widetilde{\mho} \in \mathscr{F}^{c}(G)} \prod_{g \in \mathfrak{F}}\left(-\mathbb{C}_{g}\right) G .
$$

By $\phi_{4}^{4}$-like graphs we mean graphs obeying

$($ H 3.1 $)=($ H 2.1) Roughly speaking we consider graphs built with any power

$($ H 3.2 $)=($ H 2.2) law propagators and having at most $\bar{L}$ lines hooked to

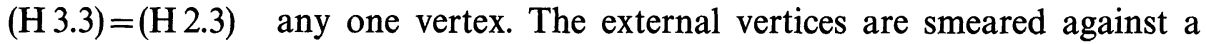
general test function.

(H 3.4) Power Counting: We assume that if $g$ is a connected subgraph of $G$ then $|E(g)| \geqq 5 \Rightarrow D(g) \leqq-4 \varepsilon E(g)$, $|E(g)|=4 \Rightarrow D(g) \leqq 0$, 
$|E(g)|=3 \Rightarrow D(g) \leqq 0$,

$|E(g)|=2 \Rightarrow D(g) \leqq \min \{2 p(\ell) \mid \ell \in E(g)\}$.

Remark. The effect of the last inequality is as follows. Take any connected biped and add to it any line of $E(g) \cap G$. The result is at worst of degree $0: D(O \longrightarrow) \leqq 0$.

We also assume that $D(g) \geqq 4 \varepsilon$ for any biped $g$ which is the closure of a proper divergent quadruped and that for non-integral positive $D(g)$ we have $D[g]+1$ $\geqq D(g)+4 \varepsilon$ where as in Sect. II $D[g]$ denotes the integer part of $D(g)$.

(H 3.5) Closed Forests. There is a constant $K^{\prime \prime}$ such that the number $\left|F^{c}(G)\right|$ of closed, proper divergent forests of $G$ is bounded by $K^{\prime \prime L(G)}$.

We remark that, for example, $\phi_{4}^{4}$ (even with lower order vertices) and Gross$\mathrm{Neveu}_{2}$ obey these hypotheses. With these hypotheses we have (using the same notation as in Theorem 2.1).

Theorem 3.1. If hypotheses $(\mathrm{H} 3.1)-(\mathrm{H} 3.5)$ are satisfied then for any $0<\zeta<1$ and any $v_{g} \in \mathfrak{B}_{E}(G)$ there exists a constant $K$ such that

$$
\text { |f } f(\mathbf{x})\left(\Re^{c} G\right)(\mathbf{x}) d \mathbf{x} \mid \leqq K^{L(G)} S^{c}(G) ! \sup _{\mathbf{j} \in T(G)}\left\|\left(\prod_{v \in \mathfrak{B}_{E}(G)} \nabla_{x_{v}}^{j_{v}}\right) f\right\|_{G, v_{G}},
$$

$S^{c}(G)$ being defined by (2.3).

Remark. Combining Theorem 3.1 with the combinatoric lemma of [CR] Appendix C, gives a global $K^{n} n$ ! bound on the $n^{\text {th }}$ order of perturbation theory for $\phi_{4}^{4}$-like models, hence the "local existence of their Borel transform" as in [CR].

Proof. It suffices to consider graphs $G$ that have no one-point subgraphs; all divergent monopeds are renormalized to zero and all others are just constants. Our principal task here is to produce a replacement for (2.7) which ensures that we still get renormalization cancellations when we need them even though $\mathfrak{F}^{c}(G)$ is a lot smaller than $\mathfrak{F}^{D}(G)$. We need to redefine $\mathfrak{S}_{\mu}$ and $\mathfrak{D}_{\mu}$, the operators which decide when subgraphs in a forest are safe or dangerous. Suppose we are trying to bound $\mathfrak{C}_{g}^{\sharp} g$. So pick any assignment of momentum scales to lines of $g$ and pick any connected subgraph $h \cong g$. Is $h$ dangerous? Of course before we get to $h$ we will have applied a $\mathbb{C}_{h^{\prime}}^{\sharp}$ for every $\mathfrak{F} \ni h^{\prime} \mathrm{C} g$. So we really need to consider $g / A_{\mathfrak{F}}(g)$.

In Sect. II, $h$ is dangerous if $h$ has $D(h) \geqq 0$ and if $h / A_{\mathscr{F}}(g)$ is a connected component of $\left(g / A_{\mathfrak{F}}(g)\right)_{j}$ for some $j$, i.e.

$$
i_{h}=\min \left\{i_{\ell} \mid \ell \in h \backslash A_{\mathscr{F}}(g)\right\}>\max \left\{i_{\ell} \mid \ell \in E(h) \cap g\right\}=e_{h} .
$$

The problem is that there are two situations in which we will be unable, by law, to renormalize $h$ even if it satisfies this criterion. We consider them in turn.

Firstly, $h$ might be 1PR. We know that this shouldn't be a problem if we have renormalized its 1PI components properly. Consider the "end" 1PI components of $h$,i.e. the components for which precisely one external line is internal of $h$. Each end can be either a single vertex $($ e.g. $\bigcirc$ ), a biped, a triped or a quadruped. Since 
$h$ is divergent (H3.4) ensures that at least one end is a proper, $D>0$ biped $h_{p}$. In addition, if you sum over all such $h_{p}$ 's associated with $h$

$$
\sum_{h_{p}} D\left(h_{p}\right) \geqq D(h)+\Delta \varepsilon .
$$

Hence as long as we can choose $v_{E}$ of (2.6) to be the one external vertex of $h_{p}$ that is not external to $h$ too, all derivatives arising from the renormalization of $h_{p}$ act on external lines of $h$ and have the same effect as renormalizing $h$ itself. In fact this choice of $v_{E}$ does not pose a problem. In Sect. II, for reasons which remain valid here, we always applied (2.6) to $h^{\prime}$ with $v_{E}$ being the fixed vertex $v_{h^{\prime}}$ of $h^{\prime}$. These fixed vertices were chosen in Module 5. But there was a lot of freedom in their choice. The only time there was a restriction was when $v_{B_{\xi^{\prime}}\left(h^{\prime}\right)}$, i.e. $v_{g}$ in our case, appeared in $h^{\prime}$. But bipeds never have external vertices in common with larger 1PI graphs. Hence it suffices to supplement Module 5 with the rule

“if $v_{B_{\xi^{\prime}}\left(h^{\prime}\right)} \notin h^{\prime}$, then set $v_{h^{\prime}}$ to be the vertex of $h^{\prime}$ which carry the line obeying $i_{\ell}=\max \left\{i_{\ell} \mid \ell \in E\left(h^{\prime}\right)\right\}$."

However we must be careful to ensure that $h_{p}$ is indeed renormalized. If we keep the same criterion as in Sect. II this need not happen. $e_{h_{p}}$ will be the scale of the external line of $h_{p}$ that is internal to $h$. It may very well be higher than $i_{h_{p}}$. Hence for bipeds we should replace $e_{h^{\prime}}$ by

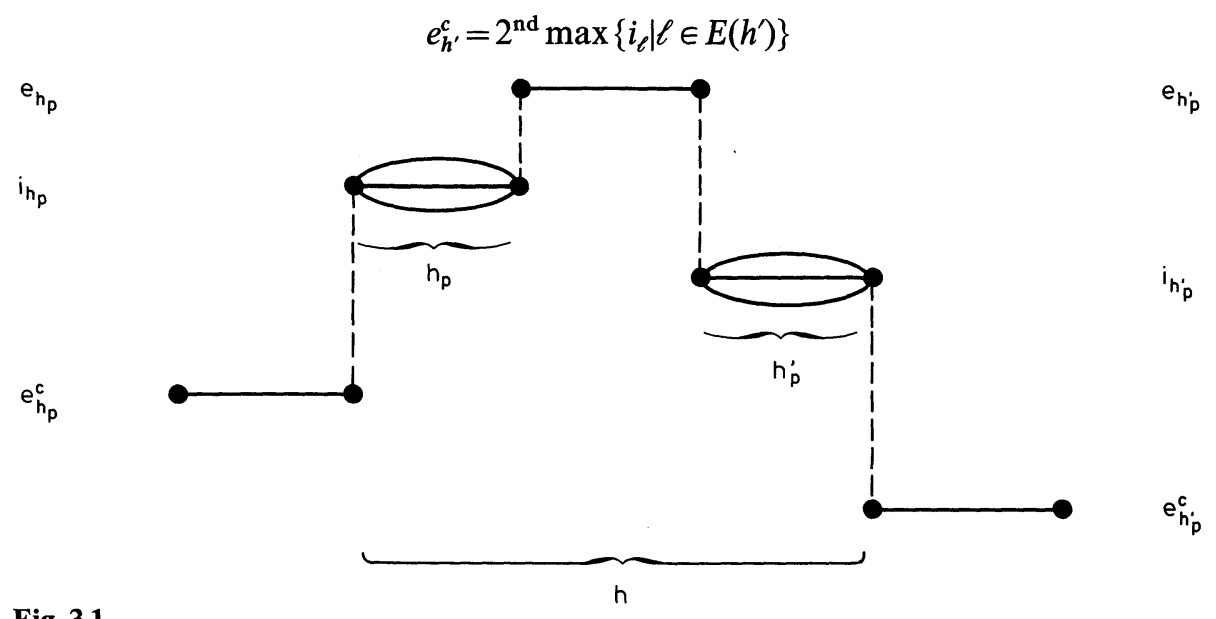

The second situation in which we will not be able to renormalize an $h$ when we want to, occurs when $h$ is proper but is not closed (see Fig. 2.1). As long its closure, the biped $h^{*}$, is renormalized this is again not a problem. Call the external vertices of $h^{*} x$ and $y$. Recall how renormalization works. We get $D\left[h^{*}\right]+1 \geqq D(h)+1$, $(x-y)$. $\nabla^{\prime}$ 's from the application of $\left(1-\widetilde{C}_{h^{*}}\right)$. Each $\nabla$ when applied to a propagator of scale $j$ produces an $M^{j}$. The decay $\exp \left\{-\right.$ const $\left.M^{i_{h^{*}|x-y|}}\right\}$ coming from propagators joining $x$ to $y$ in $h^{*}$ can be used to turn the $(x-y)$ into an $M^{-i_{h^{*}}}$. Hence we have the decay $M^{-\left(i_{h^{*-}}\right)}$ between $i_{h^{*}}$ and $j$. But $x$ and $y$ are also external vertices of $h$ so it produces the decay $M^{- \text {const } i_{h}|x-y|}$ between $x$ and $y$. Using it instead gives $M^{-\left(i_{h}-j\right)}$, just what we want for the renormalization of $h$ ! 
Fig. 3.2

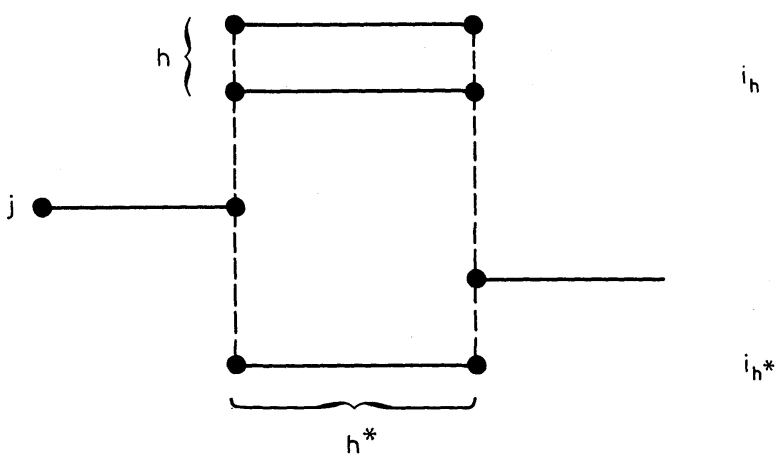

Again we must modify the definition of dangerous to ensure that $h^{*}$ is renormalized whenever $h$ is dangerous.

Here are the new definitions of $i, e, \mathfrak{S}_{\mu}$, and $\mathfrak{D}_{\mu}$. Fix any forest $\mathfrak{F} \in \mathfrak{F}^{c}(G)$ and any assignment of momenta $\mu$. First define for all $g \in \mathfrak{F}$

$$
i_{g}^{c}(\mathfrak{J})=\max _{h} \min \left\{i_{\ell} \mid \ell \in h \backslash A_{\mathfrak{F}}(g)\right\} .
$$

Here max runs over all $h$ obeying

$-h^{*}=g$,

$-h$ is compatible with $\mathfrak{F}$,

- $h / A_{\mathscr{F}}(g)$ is a proper component of $\left(g / A_{\mathfrak{F}}(g)\right)_{j}$ for some $j$. We will denote the $h$ giving the maximum $I_{g}^{c}(\mathfrak{F})$. Then define inductively from large to small in the ordering by containment of $\mathfrak{F}$

$$
e_{g}^{c}(\mathfrak{F})= \begin{cases}\max \left\{i_{\ell} \mid \ell \in E(g) \subset B_{\widetilde{S}_{\mu}(\mathfrak{F})}(g)\right\} & \text { if } g \text { is a triped or quadruped } \\ 2^{\text {nd }} \max \left\{i_{\ell} \mid \ell \in E(g)\right\} & \text { if } g \text { is a biped, }\end{cases}
$$

and

$$
\begin{array}{ll}
g \in \mathfrak{S}_{\mu}^{c}(\mathfrak{F}) \Leftrightarrow g \in \mathfrak{F}, & e_{g}^{c}(\mathfrak{F}) \geqq i_{g}^{c}(\mathfrak{F}), \\
g \in \mathfrak{D}_{\mu}^{c}(\mathfrak{F}) \Leftrightarrow g \in \mathfrak{F}, & e_{g}^{c}(\mathfrak{F})<i_{g}^{c}(\mathfrak{F}) .
\end{array}
$$

Remark. We do not demand in (3.3a) that $h$ be divergent or even a quadruped. In general a proper subgraph $h$ is closed if $h$ contains the closure of each proper quadruped contained in $h$. In general the closure $h^{*}$ of a proper subgraph is the smallest closed subgraph containing $h$. If we were to demand that $h$ be divergent $\mathfrak{S}_{\mu}^{c}$ would not have the desired properties. In particular (3.5a) (to follow) would fail. Similarly if we are to replace $B_{\Im_{\mu}^{c}(\Re)}(g)$ by $B_{\mathfrak{g}}(g)$ in $(3.3 b),(3.5 b)$ would fail.

These are essentially the same as the corresponding definitions in [CR]. [In [CR] $i_{g}^{\mathfrak{c}}(\mathfrak{F})$ is $x(g, \mathfrak{F}), e_{g}^{c}(\mathfrak{F})$ is $y(g, \mathfrak{F})$ and $\left.\mathfrak{S}_{\mu}^{c}(\mathfrak{F})=\mathfrak{S}(\mathfrak{F})\right]$. As there we have, for $g \in \mathfrak{F} \in \mathfrak{F}^{c}(G)$,

$$
\begin{aligned}
& i_{g}^{c}\left(\Im_{\mu}^{c}(\mathfrak{F}) \cup\{g\}\right)=i_{g}^{c}(\mathfrak{F}), \\
& e_{g}^{c}\left(\Im_{\mu}^{c}(\mathfrak{F}) \cup\{g\}\right)=e_{g}^{c}(\mathfrak{F}) \text {, } \\
& \mathfrak{\Im}_{\mu}^{c}\left(\mathfrak{\Im}_{\mu}^{c}(\mathfrak{F})\right)=\mathfrak{\Im}_{\mu}^{c}(\mathfrak{F}),
\end{aligned}
$$


we also have the analogue of Lemma 2.3. Define

$$
\begin{aligned}
\mathfrak{H}_{\mu}^{c}(\mathfrak{F})= & \{g \leqq G \mid g \text { is closed, proper, divergent, compatible } \\
& \text { with } \left.\mathfrak{F}, g \in \mathfrak{D}_{\mu}^{c}(\mathfrak{F} \cup\{g\})\right\} .
\end{aligned}
$$

Lemma 3.2. Let $\mathfrak{F}=\mathfrak{S}_{\mu}^{c}(\mathfrak{F})$. Then

(a) $\mathfrak{F} \cup \mathfrak{H}_{\mu}^{c}(\mathfrak{F}) \in \mathfrak{F}^{c}(G)$ and

(b) for any $\mathfrak{F}^{\prime} \in \mathfrak{F}^{c}(G)$

$$
\mathfrak{S}_{\mu}(\mathfrak{F})=\mathfrak{F} \Leftrightarrow \mathfrak{F} \subseteq \mathfrak{F}^{\prime} \cong \mathfrak{F} \cup \mathfrak{H}_{\mu}^{c}(\mathfrak{F}) .
$$

The proof is in [CR].

As a consequence of this lemma we have the analogue of (2.17) for $\Re^{c} G$ :

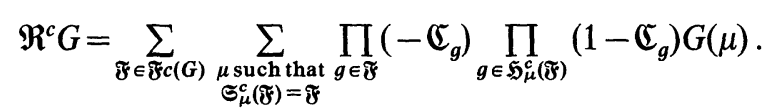

Each term in this sum will be bounded inductively with each inductive step involving an application of the procedure of Sect. II. The basis for understanding the bound is understanding which divergent diagrams are encountered and how they are dealt with. So pick any $g \in \mathfrak{F} \cup \mathfrak{H}_{\mu}^{c}(\mathfrak{F})$, and suppose we are attempting to bound $\prod_{g \supseteq g^{\prime} \in \mathfrak{F}}\left(-\mathfrak{C}_{g}\right) \prod_{g \supseteq g^{\prime} \in \mathfrak{F}_{\mathfrak{f}}^{c}(\mathfrak{F})}\left(1-\mathfrak{C}_{g}\right) g(\mu)$, having already dealt with smaller sub-

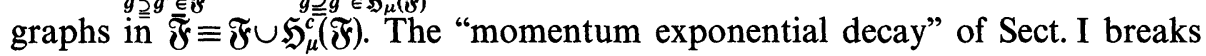
down only when we encounter a connected component $h / A_{\overline{\mathfrak{F}}}(g)$ of $\left(g / A_{\overline{\mathfrak{F}}}(g)\right)_{j}$ for which $h$ is divergent. In order for this to happen we must have either $h=g$ or $i_{h}(\overline{\mathfrak{F}})>e_{h}(\overline{\mathfrak{Z}})$, where we recall that

$$
\begin{aligned}
& i_{h}(\overline{\mathfrak{F}})=\min \left\{i_{\ell} \mid \ell \in h \backslash A_{\overline{\mathfrak{F}}}(h)\right\}, \\
& e_{h}(\overline{\mathscr{F}})=\max \left\{i_{\ell} \mid \ell \in E(h) \cap g\right\} .
\end{aligned}
$$

In the event that $h=g$ we have the situation of Sect. II; either $h \in \mathfrak{F}$ in which case we have the inequalities

$$
i_{h}(\overline{\mathscr{F}}) \leqq i_{h}^{c}(\overline{\mathscr{F}}) \leqq e_{h}^{c}(\overline{\mathscr{F}})
$$

putting a cutoff on the allowed values of $i_{h}(\overline{\mathscr{F}})$; or $h \in \mathfrak{H}_{\mu}(\mathfrak{F})$, in which case we have renormalization giving exponential decay between $i_{h}(\mathfrak{F})$ and the highest external leg differentiated. But unlike the case of Sect. II we may also have $h \subset g$. Because of the difference between $e_{h}(\overline{\mathscr{F}})$ and $e_{h}^{c}(\overline{\mathscr{F}})$ this may happen even if $h$ is divergent closed and 1PI, i.e. a legitimate candidate for renormalization. Since $g$ is 1PE $E(h) \cap g$ must contain at least two lines. Hence to allow $e_{h}(\overline{\mathfrak{F}})<e_{h}^{c}(\overline{\mathfrak{F}}), h$ must be a triped or quadruped; to keep $h$ out of $\mathfrak{G}_{\mu}(\mathfrak{F})$ we must have

$$
e_{h}(\overline{\mathfrak{F}})<i_{h}(\overline{\mathscr{F}}) \leqq i_{h}^{c}(\overline{\mathfrak{F}}) \leqq e_{h}^{c}(\overline{\mathscr{F}}),
$$

so there is again an ultraviolet cutoff on $i_{h}(\mathfrak{F})$. We will call the set of tripeds and quadrupeds for which (3.9) is satisfied $\mathfrak{H}_{\mu}(\mathscr{F})$. $h$ could also be 1PR of course. We already know that such an $h$ must have an "end" proper component $h_{p}$ which is a biped whose fixed vertex $v_{h_{p}}$ is hooked to the line of $E\left(h_{p}\right) \cap g$ carrying the highest momentum $\left[=\right.$ the one line in $\left.E\left(h_{p}\right) \cap h\right]$ and which has $D\left(h_{p}\right) \geqq D(h)$. For $h_{p}$ not to be in $\overline{\mathscr{F}}$ [i.e. not to be contracted to a point in $h / A_{\bar{F}}(g)$ ] it would have to obey

$$
e_{h_{p}}^{c}\left(\mathfrak{F} \cup\left\{h_{p}\right\}\right) \geqq i_{h_{p}}^{c}\left(\mathfrak{F} \cup\left\{h_{p}\right\}\right) \geqq i_{h_{p}}(\overline{\mathfrak{F}}) \geqq i_{h}(\overline{\mathfrak{F}})>e_{h}(\overline{\mathfrak{F}}),
$$


which is impossible. Hence $h_{p}$ will provide the exponential decay between $i_{h}(\bar{\S})$ and $e_{h}(\overline{\mathscr{F}})$ that $h$ needs.

The last way to get a divergent connected component $h / A_{\overline{\mathfrak{F}}}(g)$ of $\left(g / A_{\tilde{\mathfrak{g}}}(g)\right)_{j}$ is for $h$ to be a proper, divergent quadruped which is not closed. Then its closure, the biped $h^{*}$, will have

$$
i_{h^{*}}^{c}\left(\overline{\mathfrak{F}} \cup\left\{h^{*}\right\}\right) \geqq i_{h}(\overline{\mathscr{F}})>e_{h}(\overline{\mathscr{F}}) .
$$

$h^{*}$ must then be $g$ [or else $E\left(h^{*}\right) \subset E(h) \cap g$ forcing $h^{*}$ into $\mathfrak{S}_{\mu}(\mathfrak{F})$ ]. If $g \in \mathfrak{G}_{\mu}$, renormalization will save the day; if $g \in \mathfrak{F}$ we have an ultraviolet cutoff on $i_{h}$.

Now that we understand where the dangers lie and how to control them we are ready to proceed with the inductive bound. As usual we may fix an arbitrary family of mutually compatible disjoint forests $\mathfrak{F}, \mathfrak{H}, \mathfrak{H}$ and consider only

$$
\mathfrak{R} g=\sum_{\mu}^{c} \prod_{g \supseteq g^{\prime} \in \mathfrak{F}}\left(-\mathfrak{C}_{g}\right) \prod_{g \supseteq g^{\prime} \in \mathfrak{S}}\left(1-\mathfrak{C}_{g}\right) g(\mu)
$$

with $g \in \mathfrak{F} \cup \mathfrak{H}$ and the sum $\sum_{\mu}^{c}$ running over momentum assignments for which

$$
\mathfrak{S}_{\mu}^{c}(\mathfrak{F})=\mathfrak{F}, \quad \mathfrak{H}_{\mu}^{c}(\mathfrak{F})=\mathfrak{H}, \quad \mathfrak{R}_{\mu}(\mathfrak{F})=\mathfrak{K} .
$$

The inductive bound is then

$$
\begin{aligned}
|\mathfrak{R} g \cdot f d \mathbf{x}| \leqq & K_{1}^{L(G)} M^{D(g) e_{g}^{\epsilon}} \lambda_{\mathcal{H}^{c}(g)}\left(\bar{e}_{g}\right) \\
& \cdot \sup _{\mathbf{j} \in T(g)}\left\|\prod_{\substack{v \in \mathfrak{P}_{E}(g) \\
v \neq v_{g}}}\left\{M^{-w j_{v}^{2}}\left(\frac{\nabla_{x_{v}}}{M^{e_{g}^{c}}}\right)^{j_{v}}\right\} f\right\|_{g}^{c},
\end{aligned}
$$

where

$$
\begin{aligned}
u^{c}(g) & =|\{h \in \bar{F} \cup \mathfrak{R} \mid h \cong g\}|, \\
\bar{e}_{g} & =\max \left\{i_{\ell} \mid \ell \in E(g) \cap B_{\mathfrak{F}}(g)\right\},
\end{aligned}
$$

and $T(g)$ is as described in Sect. II. $\|\cdot\|_{g}^{c}$ is the same as $\|\cdot\|_{g}$ except that the local suprema are taken over balls of radius $M^{-\left(e_{g}^{c}+1\right)}$ and the decay factor $g_{e_{g}}$ is replaced by

$$
\begin{aligned}
g_{e_{g}}^{c}\left(\mathbf{x}_{r}\right)= & \sup _{h} \sup _{\left\{x_{v} \in \mathfrak{B}_{I}(g / \mathscr{F})\right\}} \prod_{\ell \in h / \mathscr{F}}\left\{\exp \left(-(1-\zeta) M^{e_{g}^{c}+1}\left|x_{\ell}-y_{\ell}\right|\right)\right\} \\
& \cdot \prod_{\ell \in(g \sim h) / \mathscr{F}}\left\{\exp \left(-(1-\zeta) \mid x_{\ell}-y_{\ell}\right)\right\} M^{d\left(e_{g}^{\varepsilon}+1\right)\left(V_{E}(g / \mathfrak{F})-1\right)}
\end{aligned}
$$

with the sup being over subgraphs of $g$ that are proper, consistent with $\mathfrak{F}$ and generate $g: h^{*}=g$.

We can now follow the procedure of Theorem 2.1 essentially verbatim until we are well into Module 5. It suffices to replace $e_{g}$ by $e_{g}^{c}$ and $i_{g}$ by $i_{g}^{c}$. We merely point out that the following essential requirements of our old proof are still satisfied:

- Whenever a $\frac{\nabla}{M^{e_{h}^{\ell}}}$ (or a local supremum on scale $\left.M^{e^{\ell}}\right)$ acts on a propagator $i_{\ell}$ we have $e_{h}^{c} \geqq i_{\ell}$,

- Whenever a $\nabla / M^{e_{h}^{e}}$ (or a local supremum of scale $M^{e_{h}^{c}}$ ) acts on an external line of $g, g$ is dangerous and consequently $e_{h}^{c}>e_{g}^{c}$. (This is not quite so obvious as the analogue in Sect. II was. It is proven in Lemma 3.3.) In particular we can still update the factors $e^{-w j_{v}^{2}}$ that are used to avoid factorials arising from the accumulation of many derivatives acting on a single propagator. 
- There is a decay of $\exp \left\{-\right.$ const $\left.M^{i c}\left|x_{v}-x_{v^{\prime}}\right|\right\}$ between any pair of external vertices of $g / \mathfrak{F}$. This is also not so obvious as in Sect. II, where every line in $g / \mathfrak{F}$ had a decay rate at least $M^{i_{g}}$. In the present situation we observe that there is a path joining $x_{v}, x_{v}$, in the generator $I_{g}^{c}(\mathfrak{F} \cup \mathfrak{H})$ of $g / \mathfrak{F} \cup \mathfrak{H}$ and that each propagator in this path has decay rate at least const $M^{i_{g}^{c}}$ by the definition of $i_{g}^{c}$. Furthermore this path crosses a number of generalized vertices $h \in \mathfrak{U}_{\mathfrak{F} \cup \mathfrak{S}}(g) \cap \mathfrak{T}$. We can construct, using the inductive hypothesis, a path across (a generator for each) such $h$ with decay rate const $M^{e_{h}^{c}+1}$. Since each such $h$ necessarily has an external line in $I_{g}^{c}(\mathfrak{F} \cup \mathfrak{H})$ Lemma 3.3 ensures that $e_{h}^{c} \geqq i_{g}^{c}$, and consequently that we have the desired decay between $x_{v}$ and $x_{v^{\prime}}$. As a result the extra power counting factors due to renormalization [replacing (2.36)] are

$$
\begin{aligned}
& \text { for } g \in \mathfrak{F} \text { at most } D[g] \text { additional factors of } M^{\left.e_{\mathfrak{g}}^{\mathfrak{g}}-\boldsymbol{i}_{g}^{\mathfrak{c}(\mathfrak{F}} \cup \mathfrak{H}\right)} \text {, } \\
& \text { for } g \in \mathfrak{S} \text { exactly } D[g]+1 \text { additional factors of } M^{e_{g}^{c}-i_{g}^{c}(\mathscr{F} \cup \mathfrak{H})} \text {. }
\end{aligned}
$$

To perform the integral over position space we again construct a graph $g^{\prime}$ consisting of $g$ with each subgraph in $\mathfrak{U}_{\mathfrak{F} \cup \mathfrak{H}}(g) \cap \mathfrak{F}$ collapsed to a point and each subgraph $h \in \mathfrak{U}_{\mathfrak{F} \cup \mathfrak{H}}(g) \cap \mathfrak{H}$ replaced by a tree joining the external vertices in $E(h / \mathfrak{F})$. Each line in this tree has decay rate const $M^{e_{h}^{c+1}}$, since the external vertices of $h$ are spanned by any generator of $h$. Each remaining line $\ell$ in $g / \mathfrak{F} \cup \mathfrak{G}$ has decay rate const $M^{i_{i}}$. As in Module 5 we perform the integral over position space. At this point the bound on $\left|\int \Re g \cdot f d \mathbf{x}\right|$ consists of

$$
K_{1}^{L(G)} \sup _{\mathbf{j} \in T(g)}\left\|\prod_{\substack{v \in \mathfrak{Q}_{E}(g) \\ v \neq v_{g}}}\left\{M^{-w j_{v}^{2}}\left(\frac{\nabla_{x_{v}}}{M^{e_{g}^{c}}}\right)^{j_{v}}\right\} f\right\|_{g}^{c}
$$

times the sum over all allowed momenta assignments of the momentum factors:

$$
\begin{aligned}
& -M^{-d k_{v}} \text { for each } v \neq v_{g} \text { of } g^{\prime} \\
& -M^{i_{\ell} \delta(\ell)} \text { for each line of } g / A_{\mathfrak{F} \cup \mathfrak{H}}(g) \\
& -M^{D(h) e_{h}^{c}} \lambda_{u^{c}(h)}\left(\bar{e}_{h}\right) \text { for each } h \in \mathfrak{A}_{\mathfrak{F} \cup \mathfrak{H}}(g) \\
& -M^{d\left(e_{\mathfrak{h}}^{c}+1\right)\left(V_{E}(h / \mathfrak{F})-1\right)} \text { for each } h \in \mathfrak{A}_{\mathfrak{F} \cup \mathfrak{H}}(g) \cap \mathfrak{H} \\
& -M^{\left(e_{g}^{c}-i_{g}^{c}(\mathscr{F} \cup \mathfrak{H})\right) D_{R}} \text { with } D_{R}=D[g]+1 \text { if } g \in \mathfrak{H} \\
& \quad \text { and } 0 \leqq D_{R} \leqq D[g] \text { if } g \in \mathfrak{F} .
\end{aligned}
$$

Our first task is to work these factors more or less into the standard form of (1.4)-(1.5). Using $M^{\alpha j}=\prod_{i=1}^{j} M^{\alpha}$ we can organize all the $M^{-d k_{v}}, M^{i_{\ell} \delta(\ell)}$, and $M^{d\left(e_{h}^{c}+1\right)\left(V_{E}(h / \mathfrak{F})-1\right)}$ factors into

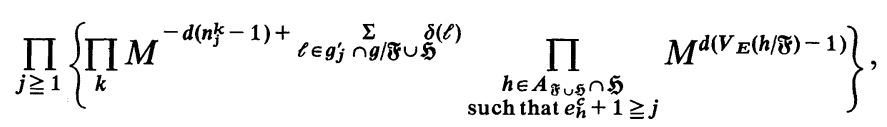

where $g_{j}^{\prime k}$ is a connected component of

$$
g_{j}^{\prime}=\left\{\ell^{\prime} \in g \mid \ell \text { has a decay rate } \geqq \text { const } M^{j}\right\}
$$

and $n_{j}^{k}$ is the number of vertices in $g_{j}^{\prime k}$. As in Module 5 for each $h \in A_{\mathfrak{F} \cup \mathfrak{H}} \cap \mathfrak{H}, j, k$, either $h \subset g_{j}^{\prime k}$ or $h$ and $g_{j}^{\prime k}$ are disjoint. In the former case we can collapse $h$ to a point with the resulting change in $n_{j}^{k}$ exactly cancelling the corresponding $M^{d\left(V_{E}(h / \mathscr{F})-1\right)}$. 
After all this collapsing is done $g_{j}^{k}$ has either become a connected component of $(g / \mathfrak{F} \cup \mathfrak{H})_{j}$ or a single point (if $g_{j}^{k}$ was exactly one $\left.h \in \mathfrak{H}_{\mathfrak{z} \cup \mathfrak{H}} \cap \mathfrak{H}\right)$. Since the latter has a factor 1 we can throw it away. Hence

$$
\text { (3.18) }=\prod_{j \geqq 1} \prod_{k} M^{D\left(\left((g / \tilde{8} \cup \mathfrak{f})_{j}^{k}\right)\right.} .
$$

In order to get something calculable we would like to use the $M^{D(h) e_{h}^{c}}$ of (3.17) to convert $D\left((g / \mathfrak{F} \cup \mathfrak{H})_{j}^{k}\right)$ into $D\left(g_{j}^{k}\right)$, where $g_{j}^{k}$ is the subgraph of $g$ consisting of $(g / \mathfrak{F} \cup \mathfrak{H})_{j}^{k}$ with all the collapsed vertices reexpanded. Now

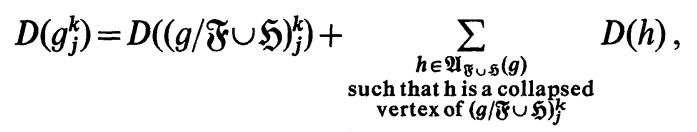

so

$$
\prod_{h \in \mathcal{Q}(\mathcal{E} \cup(g)} M^{D(h) e_{h}} \prod_{j \geqq 1} \prod_{k} M^{D\left((g / ₹ \cup \mathfrak{F})_{j}^{k}\right)}=\prod_{j \geqq 1} \prod_{k} M^{D\left(g_{j}^{k}\right)},
$$

where we recall that for $h \in \mathfrak{A}_{\mathfrak{F} \cup \mathfrak{S}}(g)$,

$$
\begin{aligned}
e_{h} & =\max \left\{i_{\ell} \mid \ell \in E(h) \cap g\right\} \\
& =\max \left\{j \mid h \text { is a collapsed vertex of some }(g / \mathfrak{F} \cup \mathfrak{H})_{j}^{k}\right\} .
\end{aligned}
$$

But we have $M^{D(h) e_{h}^{c}}$, not $M^{D(h) e_{h}}$. For tripeds and quadrupeds $D(h)=0$, so the difference between $e_{h}^{c}$ and $e_{h}$ doesn't matter. For bipeds it matters a great deal. We are now basically watching "end" quadrupeds coming to the rescue of 1PR divergent subgraphs. We write, for bipeds

$$
e_{h}^{c}=e_{h}-\left|i_{\ell_{1}}-i_{\ell_{2}}\right|
$$

where the external legs of $h$ have momentum scales $i_{\ell_{1}}, i_{\ell_{2}}$. Hence

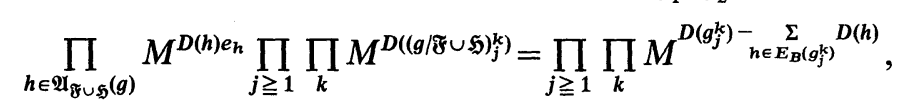

where $E_{B}\left(g_{j}^{k}\right)$ is the set of "end bipeds" of $g_{j}^{k}$, i.e. the set of bipeds $h$ such that $h$ is a collapsed vertex of $(g / \mathfrak{F} \cup \mathfrak{H})_{j}^{k}$ for which $E(h) \cap E\left((g / \mathfrak{F} \cup \mathfrak{H})_{j}^{k}\right) \neq \emptyset$.

So (3.17) has now become

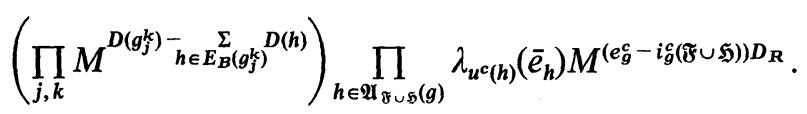

We next work on the last factor, starting with the extraction of the $M^{e_{g}^{e D(g)}}$ that we will need for the inductive bound (3.14). If $g \in \mathfrak{H}$

$$
\begin{aligned}
M^{\left(e_{g}^{c}-i_{g}^{c}\right) D_{R}} & \leqq M^{\left(e_{g}^{c}-i_{g}^{c}\right)(D(g)+4 \varepsilon)}, \text { since } D[g]+1 \geqq D(g)+4 \varepsilon \\
& \leqq\left\{\begin{array}{lll}
M^{D(g) e_{g}^{\varepsilon}} M^{-\left(i_{g}^{c}-i_{g}\right) 4 \varepsilon} M^{-i_{g} D(g)-\left(i_{g}-e_{g}^{g}\right) 4 \varepsilon} \text { if } & i_{g} \geqq e_{g}^{c} \\
M^{D(g) e_{g}^{c}} M^{-\left(i_{g}^{c}-i_{g}\right) \min (4 \varepsilon, D(g))} M^{-i_{g} D(g)} & \text { if } & e_{g}^{c}>i_{g} .
\end{array}\right.
\end{aligned}
$$

If $g \in \mathfrak{F}$, considering the cases $D_{R}=0, D_{R} \neq 0$ separately gives

$$
M^{\left(e_{g}^{\mathfrak{g}-i} \dot{c}\right) D_{R}} \leqq M^{D(g) e_{g}^{\mathfrak{c}}} M^{-\left(i_{g}^{\mathfrak{c}}-i_{g}\right) \min (D(g), 1)} M^{-i_{g} D(g)} .
$$

If $g=G$ with $D(G)<0, e_{g}^{c}=-1$ so

$$
1 \leqq M^{D(n) e_{1}^{\varepsilon}} \cdot 1 .
$$


In all of $(3.21 \mathrm{a}-\mathrm{c})$ the first factor goes to the inductive bound, the second goes to divergent proper open subgraphs and the third goes to the sum over the possible values of $i_{g}$, the lowest momentum appearing in $g / \mathfrak{F} \cup \mathfrak{H}$. Note that for any $j$ at most one $g_{j}^{k}$ can be an open proper divergent subgraph (since for $k \neq k^{\prime}, g_{j}^{k}$, and $g_{j}^{k^{\prime}}$ are disjoint) and if there is such a $g_{j}^{k}$ we must have $D\left(g_{j}^{k}\right)=0$ ( $\left(g_{j}^{k}\right.$ is a quadruped), $i_{g}<j \leqq i_{g}^{c}$ (by the definition of $i_{g}^{c}$ ) and $D(g) \geqq 5 \varepsilon$ [by ( $\left.\mathrm{H} 3.4\right)$ and the definition of open). Hence, setting

$$
\chi_{g}(j)= \begin{cases}\min (1, D(g), 1 / 4 \varepsilon) & \text { if } D(g) \geqq 0, i_{g}<j \leqq i_{g}^{c} \\ 0 & \text { otherwise }\end{cases}
$$

we have for any $j, k$

either $g_{j}^{k}$ is convergent

$$
\Rightarrow D\left(g_{j}^{k}\right)-\sum D(h)-4 \varepsilon \chi_{g}(j) \leqq D\left(g_{j}^{k}\right) \leqq-\varepsilon E\left((g / \mathfrak{F} \cup \mathfrak{H})_{j}^{k}\right) \text { by }(\mathrm{H} 3.4),
$$

or $g_{j}^{k}$ is divergent, $1 \mathrm{PR}$

$\Rightarrow D\left(g_{j}^{k}\right)-\sum D(h)-4 \varepsilon \chi_{g}(j) \leqq D\left(g_{j}^{k}\right)-\sum D(h) \leqq-4 \varepsilon \leqq-\varepsilon E\left((g / \mathfrak{F} \cup \mathfrak{H})_{j}^{k}\right)$ by $(3.2)$, or $g_{j}^{k}$ is divergent, $1 \mathrm{PI}$, open

$$
\Rightarrow D\left(g_{j}^{k}\right)-\sum D(h)-4 \varepsilon \chi_{g}(j)=-4 \varepsilon=-4 \varepsilon E\left((g / \mathfrak{F} \cup \mathfrak{H})_{j}^{k}\right),
$$

or $g_{j}^{k} \in \mathfrak{\Re}$

$$
\Rightarrow D\left(g_{j}^{k}\right)-\sum D(h)-4 \varepsilon \chi_{g}(j) \leqq 0,
$$

or $g_{j}^{k} \in \overline{\mathfrak{F}}$.

In the last case $g_{j}^{k}=g$ and $D\left(g_{j}^{k}\right)-\sum D(h)-4 \varepsilon \chi_{g}(j)=D(g)$. This happens for precisely $1 \leqq j \leqq i_{g}$, and so the resulting $\Pi M^{D\left(g_{j}^{k}\right)-\Sigma D(h)-4 \varepsilon \chi_{g}(j)}=M^{D(g) i_{g}}$ exactly cancels the $M^{-D(g) i_{g}}$ of (3.21).

So (3.17) has now become

$$
\begin{gathered}
M^{D(g) e_{g}^{\varepsilon}}\left\{\prod_{h \in \mathfrak{A} g \cup \mathfrak{g}(g)} \lambda_{u^{c}(h)}\left(\bar{e}_{h}\right)\right\} \prod_{j \geqq 1} \prod_{\substack{\text { such that } \\
g_{j}^{\prime} \varepsilon \mathcal{S} \cup \overline{\mathscr{F}}}} M^{-\varepsilon E\left(\left(g / \bar{F}^{k}\right)_{j}^{k}\right)} \\
\cdot\left\{\begin{array}{l}
1 \text { if } g \in \mathfrak{F} \text { or } D(g)<0 \text { or } g \in \mathfrak{H}, e_{g}^{c}>i_{j} \\
M^{-\left(i_{g}-e_{g}^{g}\right) 4 \varepsilon} \text { if } g \in \mathfrak{H}, i_{g} \geqq e_{g}^{c} .
\end{array}\right.
\end{gathered}
$$

As in Sect. II the next step is to move the $\lambda_{u^{c}(h)}\left(\bar{e}_{h}\right)$ 's outside the sum over momenta assignments so we can perform that sum. For each $h, \bar{e}_{h}=i_{\ell_{h}}$ for some $\ell_{h}$. If this $\ell_{h}$ is an external line of $g$ we can simply apply $\lambda_{u^{c}(h)}\left(i_{\ell_{h}}\right) \leqq \lambda_{u^{c}(h)}\left(\bar{e}_{g}\right)$. Otherwise there is a unique smallest

$$
\bar{g} \in\left\{g^{\prime} / \mathfrak{F} \cup \mathfrak{H} \mid g^{\prime} \in \mathfrak{R} \cup\{g\}, A_{\mathfrak{F} \cup \mathfrak{S}}(g) \nsubseteq g^{\prime} \cong g\right\} \equiv \mathfrak{F}_{\mathfrak{R}}
$$

in which $\ell_{h}$ appears. There is exponential decay $\left(\right.$ in $\prod_{j, k} M^{-\varepsilon E\left((g / ₹ \cup \mathfrak{F})_{j}^{k}\right)}$ ) between the scale $i_{\ell^{\prime}}$, of any $\ell^{\prime} \in \bar{g} / \mathfrak{F}_{\Re}$ and the base momentum

$$
b_{\bar{g}}=\min \left\{i_{\ell^{\prime}} \mid \ell^{\prime} \in \bar{g} / \mathfrak{F}_{\Omega}\right\}
$$


of $\bar{g}$. Using three quarters of this decay we can bound all of the $\lambda_{u^{c}(h)}$ 's associated with any one $\bar{g} \in \mathfrak{F}_{\boldsymbol{R}}$ by

$$
\begin{aligned}
M^{-\frac{3}{4} \varepsilon\left(\max _{h} i_{\ell_{h}}-b_{\bar{g}}\right)} \prod_{h} \lambda_{u^{c}(h)}\left(i_{\ell_{h}}\right) \leqq & M^{-\frac{3}{4}\left(\max _{h} i_{\ell_{h}}-b_{\bar{g}}\right)} \lambda_{\Sigma_{h} u^{c}(h)}\left(\max _{h} i_{\ell_{h}}\right) \\
& \text { by Lemma } 2.4(\mathrm{c})(\mathrm{f}) \\
\leqq & \lambda_{\bar{h}} u^{c}(h) \\
& \left(b_{\bar{g}}\right) \text { by Lemma } 2.4(\mathrm{e})
\end{aligned}
$$

Now $b_{\bar{g}}$ is in fact exactly $i_{g^{\prime}}$, the lowest momentum scale of lines in $g^{\prime} / \mathfrak{F} \cup \mathfrak{H}$, where $g^{\prime}$ is the element of $\Re \cup\{g\}$ associated with $\bar{g}$. If $g^{\prime} \in \mathfrak{R}(2.9)$ ensures that $i_{g^{\prime}} \leqq e_{g^{\prime}}^{c}(\overline{\mathfrak{F}})$ and furthermore that the line of $E\left(g^{\prime}\right)$ for which $i_{\ell}=e_{g^{\prime}}^{c}(\overline{\mathscr{F}})$ is in $E(g) \cap B_{\mathfrak{F}}(g)$ too. So $e_{g^{\prime}}^{c}(\bar{\mho}) \leqq \bar{e}_{g}$ and

$$
\lambda_{\Sigma_{h} u^{c}(h)}\left(b_{\bar{g}}\right) \leqq \lambda_{\Sigma_{n} u^{c}(h)}\left(\bar{e}_{g}\right) .
$$

If $g^{\prime}=g$ and $g \in \mathfrak{F}, b_{\bar{g}}=i_{g} \leqq i_{g}^{c} \leqq e_{g}^{c} \leqq \bar{e}_{g}$, and we again have (3.23). If $g^{\prime}=g$ and $g \in \mathfrak{H} \cup\{G\}$, we either have $i_{g} \leqq e_{g}^{c} \leqq \bar{e}_{g}$; we take three quarters of the decay $M^{-\left(i_{g}-e_{g}^{c}\right) 4 \varepsilon}$ of $(2.23)$ (or if $g=G$ with $D(G) \neq 0$ from the $\prod_{j, k} M^{-\varepsilon E\left((g / \mathfrak{z} \cup \mathfrak{G})_{j}^{k}\right)}$ ) and again apply Lemma 2.4(f):

$$
\exp \left(-\frac{3}{4} \varepsilon\left(i_{g}-e_{g}^{c}\right)\right) \lambda_{\sum_{n} u^{c}(h)}\left(i_{g}\right) \leqq \lambda_{\sum_{n} u^{c}(h)}\left(e_{g}^{c}\right) \leqq \lambda_{\Sigma_{n} u^{c}(h)}\left(\bar{e}_{g}\right)
$$

We have by now worked (3.17) into the form

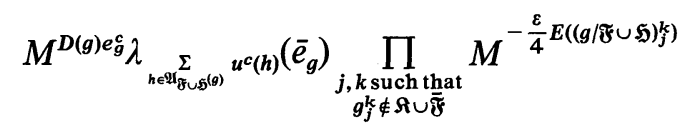

$$
\begin{aligned}
& \cdot \begin{cases}1 & \text { if } \quad g \in \mathfrak{F} \text { or } g=G, D(G)<0 \text { or } g \in \mathfrak{H}, e_{g}^{c}>i_{g} \\
M^{-\left(i_{g}-e_{g}^{c}\right) \varepsilon} & \text { if } \quad g \in \mathfrak{H}, e_{g}^{c} \leqq i_{g},\end{cases}
\end{aligned}
$$

and the last remaining problem is to perform the sum over allowed momentum assignments.

We first sum over all allowed momentum assignments with the base momenta $b_{\bar{g}}$ of all the $\bar{g} \in \mathfrak{F}_{\mathcal{A}}$ held fixed. As we have already observed every $\ell \in g / \mathfrak{F} \cup \mathfrak{H}$ appears in exactly one $\bar{g} / \mathfrak{F}_{\mathfrak{\Omega}}$. Furthermore our

$$
\prod_{j \geqq i_{g}} \prod_{k \text { such that } g_{j}^{k} \notin \cup \cup \tilde{F}} M^{-\frac{\varepsilon}{4} E\left((g / \mathfrak{Z} \cup \mathfrak{S})_{j}^{k}\right)}
$$

provides the usual momentum decay to all the $\bar{g} / \mathfrak{F}_{\Re}$ 's: for every vertex $v$ of any $\bar{g} / \mathfrak{F}_{\mathcal{A}}$ and each line entering that vertex, there is the decay factor $M^{-\varepsilon\left(i_{v}-i_{\ell}\right)}$, where $i_{v}$ is the scale of the highest line of $\bar{g} / \mathfrak{F}_{\mathcal{A}}$ entering $v$. The only catch is that, unless $\bar{g}=G$ with $D(G)<0$, there is no corresponding factors for lines $\ell$ which are in $E\left(\bar{g} / \mathfrak{F}_{\mathcal{R}}\right)$. Still as long as $b_{\bar{g}}$ is held fixed, to break the translation invariance, we can perform the sum over momentum assignments at the cost of a constant per line of $g / \overline{\mathfrak{F}}$. That leaves the sum over all possible values of the $b_{\bar{g}}$ 's. F'sr $\bar{g}=g^{\prime} / \bar{F}$ with $g^{\prime} \in \Re \cup \mathfrak{F}$ the sum is bounded by the number of values $b_{\bar{g}}$ can take. As we have already noticed $b_{\bar{g}}=i_{g^{\prime}} \leqq \bar{e}_{g}$, so each such sum produces a factor of $\bar{e}_{g}+1$. For $g^{\prime}=g \in \mathfrak{H}$ we use up the remaining $M^{-\left(i_{g}-e_{g}^{c}\right) \varepsilon}$ :

$$
\sum_{i_{g}<e_{g}^{c}} 1+\sum_{i_{g} \geqq e_{g}^{c}} M^{-\left(i_{g}-e_{g}^{c}\right) \varepsilon} \leqq \text { const }+e_{g}^{c} .
$$


Hence the sum of (3.17) over all allowed momentum assignments is bounded (aside from irrelevant constants) by

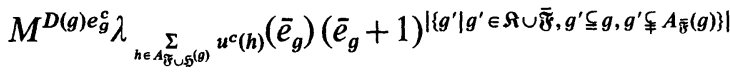

$$
\begin{aligned}
& \leqq M^{D(g) e_{g}^{\mathrm{c}}} \lambda_{u^{c}(g)}\left(\bar{e}_{g}\right) \text { by Lemma (2.4d). }
\end{aligned}
$$

That verifies the inductive bound.

Lemma 3.3. Let $h, g \in \mathfrak{F}^{\prime} \in \mathfrak{F}^{c}(G)$ with $h \in A_{\mathfrak{F}^{\prime}}(g)$.

(a) If $E(h) \cap\left\{E(g) \cup I_{g}^{c}\left(\mathfrak{F}^{\prime}\right)\right\} \neq \emptyset$, then $e_{h}^{c}\left(\mathfrak{F}^{\prime}\right) \geqq i_{g}^{c}\left(\mathfrak{F}^{\prime}\right)$.

(b) If in addition $g \in \mathfrak{D}_{\mu}\left(\mathfrak{F}^{\prime}\right)$, then $e_{h}^{c}\left(\mathfrak{F}^{\prime}\right)>e_{g}^{c}\left(\mathfrak{F}^{\prime}\right)$.

Proof. (a) $h$ has at least a vertex in common with $I_{g}^{c}\left(\mathfrak{F}^{\prime}\right)$, but cannot contain all of $I_{g}^{c}\left(\mathfrak{F}^{\prime}\right)$, since $h^{*}=h \subset g$. Since $I_{g}^{c}\left(\mathfrak{F}^{\prime}\right)$ is compatible with $\mathfrak{F}^{\prime}$ we must then have $h \subset I_{g}^{c}\left(F^{\prime}\right)$. But $I_{g}^{c}\left(F^{\prime}\right)$ is proper so

$$
2 \leqq\left|E(h) \cap I_{g}^{c}\left(\mathfrak{F}^{\prime}\right)\right|=\left|E(h) \cap I_{g}^{c}\left(\mathfrak{F}^{\prime}\right) / A_{\mathfrak{F}^{\prime}}(g)\right|,
$$

which in turn implies

$$
\begin{aligned}
i_{g}^{c}\left(\mathfrak{F}^{\prime}\right) & =\min \left\{i_{\ell} \mid \ell \in I_{g}^{c}\left(\mathfrak{F}^{\prime}\right) / A_{\mathfrak{F}^{\prime}}(g)\right\} \\
& \leqq \min \left\{i_{\ell} \mid \ell \in E(h) \cap I_{g}^{c}\left(\mathfrak{F}^{\prime}\right) / A_{\mathfrak{F}^{\prime}}(g)\right\} \\
& \leqq 2^{\text {nd }} \max \left\{i_{\ell} \mid \ell \in E(h) \cap I_{g}^{c}\left(\mathfrak{F}^{\prime}\right) / A_{\mathfrak{F}^{\prime}}(g)\right\} \\
& \leqq 2^{\text {nd }} \max \left\{i_{\ell} \mid \ell \in E(h) \cap g\right\} \leqq e_{h}^{c}\left(\mathfrak{F}^{\prime}\right) .
\end{aligned}
$$

(b) If $g \in \mathfrak{D}_{\mu}\left(\mathfrak{F}^{\prime}\right)$ we also have $i_{g}^{c}\left(\mathfrak{F}^{\prime}\right)>e_{g}^{c}\left(\mathfrak{F}^{\prime}\right)$.

\section{References}

[CR] de Calan, C., Rivasseau, V.: Local existence of the Borel transform in Euclidean $\phi_{4}^{4}$. Commun. Math. Phys. 82, 69 (1981)

[CPR] de Calan, C., Petritis, D., Rivasseau, V.: Preprint, Ecole Polytechnique (January 1985)

[FMRS1] Feldman, J., Magnen, J., Rivasseau, V., Sénéor, R.: Bounds on completely convergent Euclidean Feynman graphs. Commun. Math. Phys. 98, 273-288 (1985)

[FMRS2] Feldman, J., Magnen, J., Rivasseau, V., Sénéor, R.: Méthodes pour la théorie constructive des champs renormalisables asymptotiquement libres, Proceedings of RCP 25, Strasbourg, June 1984

[FMRS3] Feldman, J., Magnen, J., Rivasseau, V., Sénéor, R.: The infrared behavior of $\phi_{4}^{4}$ (in preparation)

[G] Gallavotti, G.: Renormalization theory and ultraviolet stability for scalar fields via renormalization group methods. Preprint, University of Roma II

[GN1] Gross, D., Neveu, A.: Dynamical symmetry breaking in asymptotically free-field theories. Phys. Rev. D 10, 3235 (1974)

[GN2] Gallavotti, G., Nicolò, F.: Renormalization theory in four dimensional scalar fields. I, II. Commun. Math. Phys. (to appear)

[L] Lautrup, B.: On high order estimates in QED. Phys. Lett. 69B, 109 (1977)

Communicated by A. Jaffe

Received November 1, 1984; in revised form January 24, 1985 
